\title{
THE FORMATION AND DYNAMICAL EVOLUTION OF YOUNG STAR CLUSTERS
}

\author{
M. S. FujII ${ }^{1,3,4}$ and S. Portegies ZWART ${ }^{2}$ \\ ${ }^{1}$ Division of Theoretical Astronomy, National Astronomical Observatory of Japan, 2-21-1 Osawa, Mitaka, Tokyo 181-8588, Japan \\ ${ }^{2}$ Leiden Observatory, Leiden University, P.O. Box 9513, 2300 RA, Leiden, The Netherlands; michiko.fujii@nao.ac.jp \\ Received 2015 April 30; accepted 2015 November 26; published 2016 January 18
}

\begin{abstract}
Recent observations have revealed a variety of young star clusters, including embedded systems, young massive clusters, and associations. We study the formation and dynamical evolution of these clusters using a combination of simulations and theoretical models. Our simulations start with a turbulent molecular cloud that collapses under its own gravity. The stars are assumed to form in the densest regions in the collapsing cloud after an initial free-fall time of the molecular cloud. The dynamical evolution of these stellar distributions is continued by means of direct $\mathrm{N}$-body simulations. The molecular clouds typical of the Milky Way Galaxy tend to form embedded clusters that evolve to resemble open clusters. The associations were initially considerably more clumpy, but they lost their irregularity in about a dynamical timescale, due to the relaxation process. The densest molecular clouds, which are absent in the Milky Way but are typical in starburst galaxies, form massive, young star clusters. They indeed are rare in the Milky Way. Our models indicate a distinct evolutionary path from molecular clouds to open clusters and associations or to massive star clusters. The mass-radius relation for both types of evolutionary tracks excellently matches the observations. According to our calculations, the time evolution of the half-mass-radius relation for open clusters and associations follows $r_{\mathrm{h}} / \mathrm{pc}=2.7\left(t_{\mathrm{age}} / \mathrm{pc}\right)^{2 / 3}$, whereas for massive star clusters $r_{\mathrm{h}} / \mathrm{pc}=0.34\left(t_{\mathrm{age}} / \mathrm{Myr}\right)^{2 / 3}$. Both trends are consistent with the observed age-mass-radius relation for clusters in the Milky Way.
\end{abstract}

Key words: galaxies: star clusters: general - ISM: clouds - methods: numerical - open clusters and associations: general

\section{INTRODUCTION}

Star clusters are classically categorized into two groups: Galactic open clusters and globular clusters. Open clusters are generally rather young $(\lesssim 1 \mathrm{Gyr})$ with typically $100-10^{4}$ stars; hereafter we call them "classical" open clusters. Globular clusters are old $(\gtrsim 10 \mathrm{Gyr})$, more massive $\left(\gtrsim 10^{5} M_{\odot}\right)$, and dense $\left(\gtrsim 100 M_{\odot} \mathrm{pc}^{-3}\right)$. Recent observations indicate that there is a wide variety among open star clusters in the Milky Way. These types include

1. embedded clusters, which are very young, $\lesssim 3 \mathrm{Myr}$, and therefore still embedded in their natal gas cloud (Lada \& Lada 2003); embedded clusters reside in the Galactic disk and are composed of several 100 stars in a volume with a radius of $\sim 1$ pc (Figuerêdo et al. 2002);

2. associations, which are considered unbound from the moment they were born (Gieles \& Portegies Zwart 2011); and

3. young massive clusters, which are also young $(\lesssim 10 \mathrm{Myr})$ and extremely dense $\left(\gtrsim 10^{3} M_{\odot} \mathrm{pc}^{-3}\right.$ ) (Portegies Zwart et al. 2010).

Some of the embedded clusters evolve into classical open clusters if they survive gas expulsion (Lada \& Lada 2003; Fujii 2015a).

Young massive clusters are common in nearby starburst galaxies, such as in M83 (Bastian et al. 2011) and M51 (Chandar et al. 2011), but they are rare in the Milky Way. Two young massive star clusters reside close to the Galactic center, namely,

\footnotetext{
${ }^{3}$ NAOJ Fellow.

4 Current address: Department of Astronomy, Graduate School of Science, The University of Tokyo, 7-3-1 Hongo, Bunkyo-ku, Tokyo, 113-0033, Japan; fujii@astron.s.u-tokyo.ac.jp
}

Arches and Quintuplet, and the others are in the spiral arms. This latter category includes the clusters NGC 3603, Westerlund 1 and 2, and Trumpler 14 (Portegies Zwart et al. 2010).

Pfalzner (2009) suggested another type of young star clusters: "leaky clusters." Leaky clusters have a mass similar to those of the massive clusters $\left(\sim 10^{4} M_{\odot}\right)$, but with a much lower density $\left(\sim 1-10 M_{\odot} \mathrm{pc}^{-3}\right)$. Portegies Zwart et al. (2010) classified the leaky clusters listed in Pfalzner (2009) as OB associations.

According to the arguments in Gieles \& Portegies Zwart (2011), the distinction between an open cluster and an association can be made on the ratio between the age of the stars and the dynamical time of the system $\left(t_{\text {age }} / t_{\mathrm{dyn}}\right)$. If the ages of the stars exceed the dynamical age of the system, the stars must be bound together. Otherwise the system is unbound.

In an attempt to clarify the various classes and families of stellar conglomerates, we discuss in this paper the formation and dynamical evolution of young star clusters by means of simulations. The numerical modeling used here allows us to make a more clear distinction between the difference in initial conditions and the difference in evolution. It therefore helps us to differentiate between the various classes and families of clustered stellar environments.

In previous papers, we performed direct $N$-body simulations using initial conditions constructed from the results of hydrodynamical simulations of turbulent molecular clouds. There we found that young massive clusters form from turbulent molecular clouds if the local star-formation efficiency (SFE) depends on the local gas density (Fujii 2015b; Fujii \& Portegies Zwart 2015). We also found that observed embedded clusters tend to evolve into classical open clusters (Fujii 2015b). Our simulations, however, did not provide a channel for forming associations (or leaky clusters, according to Pfalzner 2009). 
At this point it is still unclear how leaky clusters form. Pfalzner (2011) proposed that leaky clusters are born as embedded clusters, that their mass increases due to a prolonged phase of star formation, and that the expansion is driven by the expulsion of the residual gas. This scenario was tested by means of simulations in Pfalzner \& Kaczmarek (2013), Parmentier \& Pfalzner (2013), and Pfalzner et al. (2014), in which it was concluded that the known embedded clusters in the Galactic disk are the ancestors of leaky clusters.

In our previous simulations we did not find leaky clusters. This may have been a result of our selected initial conditions for the parental molecular cloud, for which we chose rather massive $\left(10^{5}-10^{6} M_{\odot}\right)$ and dense $\left(100-1000 \mathrm{~cm}^{-3}\right)$ structures. The molecular clouds observed in the Milky Way tend to follow Larson's relation (Larson 1981), which indicates a relation between cloud mass and density: according to this relation, massive clouds have a lower density if the clouds are close to being virialized. The initial conditions in our previous study would then be biased toward too-dense clouds compared to the typical massive clouds in the Milky Way.

In this paper, we expand on the initial parameter space by also allowing massive clouds with a lower density. This expansion of the parameter space helps in the formation of associations, as well as in making dense, massive clusters. We support our numerical models with theoretical arguments in order to understand the dynamical evolution of each type of star clusters (classical open, embedded, young massive, and leaky clusters or associations).

\section{SIMULATIONS}

We perform a series of $N$-body simulations based on the results of hydrodynamical simulations of turbulent molecular clouds. We first perform simulations of molecular clouds with a turbulent velocity field using a smoothed particle hydrodynamics (SPH) code. The resolution of the hydrodynamical simulations is relatively low, and therefore the simulation cannot resolve the formation of individual stars, but can resolve the clumpy structures of the gas. After around one free-fall time of the initial molecular clouds, we stop the hydrodynamical simulations and replace a portion of the gas particles with stellar particles assuming an SFE depending on the local density. We then remove all residual gas particles and perform direct $N$-body simulations only with stellar particles. We describe the details of the initial conditions and the simulations in the following (see also Fujii 2015b; Fujii \& Portegies Zwart 2015).

\subsection{The Astronomical Multipurpose Software Environment}

The hydrodynamical simulations and the data analyses in this study are performed using the AMUSE framework (Pelupessy et al. 2013; Portegies Zwart et al. 2013). AMUSE is not a single code, but an extensive library of more than 50 high-performance simulation codes. The AMUSE consortium is a spin-off from the modeling and observing DEnse STellar systems (MODEST) community, which after three workshops in Lund, Amsterdam, and Split culminated in a first implementation of what at that time was called the Multi-user Software Environment (or MUSE) (Portegies Zwart et al. 2009). Later the package was extended from its primary objective of Noah's Arc (two codes per domain) to about a dozen codes per domain.
Apart from scientific production software, AMUSE also supports generating the initial conditions for data processing. The fundamental package is written in the Python language, and it is freely available via Github and via the project web page at http://amusecode.org. All the scripts used to run the simulations in this paper are available via this project web page.

\subsection{Hydrodynamical Simulations}

\subsubsection{Initial Conditions for Molecular Clouds}

All initial conditions are generated using the AMUSE framework. We adopt isothermal $(30 \mathrm{~K})$ homogeneous spheres as initial conditions of molecular clouds following Bonnell et al. (2003). We give a divergence-free, random Gaussian velocity field $\delta \boldsymbol{v}$ with a power spectrum $|\delta v|^{2} \propto k^{-4}$ (Ostriker et al. 2001; Bonnell et al. 2003). The spectral index of -4 appears in the case of compressive turbulence (Burgers turbulence), and recent observations of molecular clouds (Heyer \& Brunt 2004) and numerical simulations (Federrath et al. 2010; Roman-Duval et al. 2011; Federrath 2013a) also suggested values similar to -4 . Each model is run with a different random seed for a realization of the initial conditions.

We adopt the virial ratio $\left|E_{\mathrm{k}}\right| /\left|E_{\mathrm{p}}\right|=1$ (here $E_{\mathrm{k}}$ and $E_{\mathrm{p}}$ are kinetic and potential energies) and three masses for the molecular clouds of $M_{\mathrm{g}}=10^{4}, 4 \times 10^{5}$, and $10^{6} M_{\odot}$. The densities of these molecular clouds are $\rho_{\mathrm{g}}=17,170$, and $1700 \mathrm{~cm}^{-3}$ (which corresponds to 1,10 , and $100 M_{\odot} \mathrm{pc}^{-3}$ assuming that the mean weight per particle is $2.33 \mathrm{~m}_{\mathrm{H}}$, respectively). The initial conditions are summarized in Table 1.

Once we chose the cloud mass and density, the radius $\left(R_{\mathrm{g}}\right)$ and the velocity dispersion in three dimensions $\left(\sigma_{\mathrm{g}}\right)$ are determined. Some of our models (such as models m1M-d1-s15, m1M-d1-s16, and m1M-d1-s17, with $M_{\mathrm{g}}=10^{6} M_{\odot}$ and $\rho_{\mathrm{g}}=17 \mathrm{~cm}^{-3}$ ) roughly follow Larson's relation (Larson 1981):

$$
\sigma \sim\left(\frac{L}{1 \mathrm{pc}}\right)^{0.5}\left(\mathrm{~km} \mathrm{~s}^{-1}\right)
$$

where $\sigma$ is the velocity dispersion, and $L$ is the size of the cloud (Heyer \& Brunt 2004; Mac Low \& Klessen 2004).

In Figure 1 we present the distribution of mass and density for the simulations listed in Table 1 . In order to determine the mass of a molecular cloud that is consistent with Larson's relation, we adopt a velocity dispersion of $\sigma_{\mathrm{g}} \simeq \sqrt{G M_{\mathrm{g}} / R_{\mathrm{g}}}$. Some models initially have a higher velocity dispersion, which we motivate through cloud-cloud collisions (Furukawa et al. 2009; Fukui et al. 2013, 2014) or to simulate molecular clouds in starburst galaxies. We further motivate and discuss our choice of the initial conditions in Section 4.

\subsubsection{SPH Simulations}

We perform hydrodynamical simulations using the SPH code Fi (Hernquist \& Katz 1989; Gerritsen \& Icke 1997; Pelupessy et al. 2004; Pelupessy 2005) in the AMUSE framework. Our calculations have a relatively low mass resolution of $m=1 M_{\odot}$ per particle. The gravitational softening length during the hydrodynamical simulations is $0.1 \mathrm{pc}$, and the SPH softening length $(h)$ is chosen such that $\rho_{\mathrm{g}} h^{3}=m N_{\mathrm{nb}}$ (Springel \& Hernquist 2002). Here $N_{\mathrm{nb}}=64$ is the target number of neighbor particles. With the adopted isothermal gas temperature of $30 \mathrm{~K}$ we can resolve the Jeans 
Table 1

Initial Conditions for the Hydrodynamical Simulations

\begin{tabular}{|c|c|c|c|c|c|}
\hline Model & $\begin{array}{c}\text { Mass } \\
M_{\mathrm{g}}\left(M_{\odot}\right)\end{array}$ & $\begin{array}{l}\text { Radius } \\
r_{\mathrm{g}}(\mathrm{pc})\end{array}$ & $\begin{array}{c}\text { Density } \\
\rho_{\mathrm{g}}\left(\mathrm{cm}^{-3}\right)\end{array}$ & $\begin{array}{c}\text { Velocity Dispersion } \\
\sigma_{\mathrm{g}}\left(\mathrm{km} \mathrm{s}^{-1}\right)\end{array}$ & $\begin{array}{c}\text { Initial Free-fall Time } \\
t_{\mathrm{ff}, \mathrm{i}}(\mathrm{Myr})\end{array}$ \\
\hline m1M-d100-s7 & $1 \times 10^{6}$ & 13.4 & $1.7 \times 10^{3}$ & 19.6 & 0.81 \\
\hline m1M-d1-s15 & $1 \times 10^{6}$ & 62 & 17 & 9.1 & 8.1 \\
\hline $\mathrm{m} 1 \mathrm{M}-\mathrm{d} 1-\mathrm{s} 17$ & $1 \times 10^{6}$ & 62 & 17 & 9.1 & 8.1 \\
\hline $\mathrm{m} 400 \mathrm{k}-\mathrm{d} 100-\mathrm{s} 1$ & $4 \times 10^{5}$ & 10 & $1.7 \times 10^{3}$ & 14.4 & 0.82 \\
\hline $\mathrm{m} 400 \mathrm{k}-\mathrm{d} 100-\mathrm{s} 2$ & $4 \times 10^{5}$ & 10 & $1.7 \times 10^{3}$ & 14.4 & 0.82 \\
\hline m400k-d10-s9 & $4 \times 10^{5}$ & 21 & 170 & 9.9 & 2.5 \\
\hline m10k-d100-s4 & $1 \times 10^{4}$ & 2.87 & $1.7 \times 10^{3}$ & 4.2 & 0.81 \\
\hline m10k-d100-s5 & $1 \times 10^{4}$ & 2.87 & $1.7 \times 10^{3}$ & 4.2 & 0.81 \\
\hline m10k-d100-s6 & $1 \times 10^{4}$ & 2.87 & $1.7 \times 10^{3}$ & 4.2 & 0.81 \\
\hline m10k-d10-s11 & $1 \times 10^{4}$ & 6.2 & 170 & 2.9 & 2.6 \\
\hline m10k-d10-s12 & $1 \times 10^{4}$ & 6.2 & 170 & 2.9 & 2.6 \\
\hline
\end{tabular}

Note. All models are supervirial, with $\left|E_{\mathrm{k}}\right| /\left|E_{\mathrm{p}}\right|=1$. Here, "s" indicates the random seeds for the turbulence.

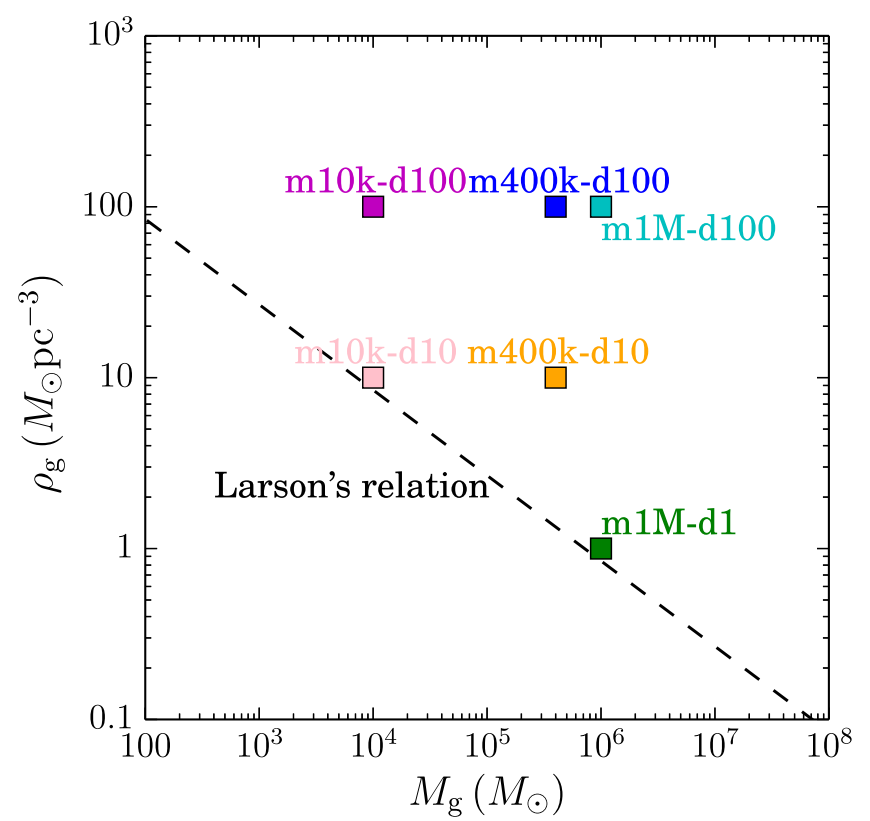

Figure 1. Mass-density relation of our initial models (see Table 1). The dashed line indicates the mass-density relation from Larson's relation (Larson 1981) for a virialized cloud $\left(\sigma_{\mathrm{g}}^{2}=G M_{\mathrm{g}} / R_{\mathrm{g}}\right)$.

instability down to $h \sim 0.4 \mathrm{pc}$, which is smaller than the typical size of known embedded clusters (1 pc) (Lada \& Lada 2003) but somewhat larger than the observed typical width of gas filaments $(\sim 0.1 \mathrm{pc})$ (Arzoumanian et al. 2011). With these limitations, we obviously cannot resolve the formation of individual stars, but we do resolve dense gas clumps. We think that the limited resolution of our hydrodynamical simulations does not pose a serious problem because we are interested in the global dynamical structure of the molecular cloud after only about an initial free-fall timescale, $t_{\mathrm{ff}, \mathrm{i}}$ (see Table 1 for the freefall timescales for each of the initial models). In fact, after $0.9 t_{\mathrm{ff}, \mathrm{i}}$ we stop the hydrodynamical simulation to analyze the resulting gas distribution, initialize stars, and continue the simulations using a gravitational $N$-body code.

\subsection{Star Formation}

After stopping the hydrodynamical simulation (around $\sim 0.9 t_{\mathrm{ff}, \mathrm{i}}$ ), we replace some of the SPH particles with stellar particles. The selection of SPH particles is based, through the local gas density $\rho$, on the local SFE $\epsilon_{\text {loc }}$ :

$$
\epsilon_{\mathrm{loc}}=\alpha_{\mathrm{sfe}}\left(\frac{\rho}{100 M_{\odot} \mathrm{pc}^{-3}}\right)^{0.5}
$$

Here $\alpha_{\text {sfe }}$ is a free parameter in our simulations to control the SFE. The form of $\epsilon_{\mathrm{loc}}$ (Equation (2)) is motivated by the observations of individual molecular clouds for which the starformation rate is argued to scale with the local free-fall timescale (Krumholz et al. 2012; Federrath 2013b).

Here we adopt $\alpha_{\text {sfe }}=0.02$, which reproduces the observed global SFE across an entire molecular cloud of several percent, but also leads to a $10 \%-30 \%$ SFE in dense regions $\left(>1000 M_{\odot} \mathrm{cm}^{-3}\right)$ (Lada \& Lada 2003; Higuchi et al. 2009; Federrath \& Klessen 2013). In Table 2 we present the global $\operatorname{SFE}(\epsilon)$ and the SFE for the dense regions $\left(\epsilon_{\mathrm{d}}\right)$ in our simulations.

Depending on the local SFE, we replace individual gas particles with individual stellar particles, conserving their positions and velocities. For each selected particle, we assign a mass from the Salpeter mass function (Salpeter 1955) between $0.3 M_{\odot}$ and $100 M_{\odot}$, irrespective of the mass of its parent $\mathrm{SPH}$ particle. The mean mass of the adopted mass function is $1 M_{\odot}$, which corresponds to the mass of individual SPH particles. Mass in our simulations is therefore globally conserved, but not locally.

\subsection{N-body Simulations}

After the stellar particles are initialized (mass randomly from the Salpeter mass function, and position and velocity from the parent SPH particle), we remove the residual gas, leaving only the stellar particles in the simulations. The instantaneous removal of the gas does not have a dramatic effect on the stellar distribution because most stars are formed in the densest regions where little low-density (residual) gas is present. The 
Table 2

Models for $N$-body Simulations

\begin{tabular}{|c|c|c|c|c|c|}
\hline Model & $\begin{array}{c}\text { Mass } \\
M_{\mathrm{s}}\left(M_{\odot}\right) \\
\end{array}$ & $\begin{array}{c}N \text { of Particles } \\
N_{\mathrm{s}} \\
\end{array}$ & $\begin{array}{c}\text { Virial Ratio }^{\mathrm{a}} \\
\left|E_{\mathrm{k}}\right| /\left|E_{\mathrm{p}}\right|\end{array}$ & $\begin{array}{c}\text { SFE (Global) } \\
\epsilon\end{array}$ & $\begin{array}{c}\text { SFE (Dense) } \\
\epsilon_{\mathrm{d}}\end{array}$ \\
\hline m1M-d100-s7 & $1.1 \times 10^{5}$ & 109080 & 0.9 & 0.11 & 0.27 \\
\hline m1M-d1-s16 & $1.9 \times 10^{4}$ & 18760 & 0.50 & 0.019 & 0.63 \\
\hline m1M-d1-s16-t0.75 & $4.6 \times 10^{3}$ & 4566 & 19 & 0.0046 & 0.42 \\
\hline m1M-d1-s16-t0.65 & $3.9 \times 10^{3}$ & 3855 & 131 & 0.0039 & 0.083 \\
\hline m1M-d1-s15-t0.75 & $5.9 \times 10^{3}$ & 5902 & 1.6 & 0.0059 & 0.49 \\
\hline m1M-d1-s15-t0.65 & $4.0 \times 10^{3}$ & 3954 & 80 & 0.0040 & 0.12 \\
\hline m1M-d1-s17-t0.75 & $5.5 \times 10^{3}$ & 5506 & 6.4 & 0.0055 & 0.26 \\
\hline m1M-d1-s17-t0.65 & $4.3 \times 10^{3}$ & 4322 & 63 & 0.0043 & 0.088 \\
\hline m400k-d100-s1 & $3.2 \times 10^{4}$ & 31895 & 1.3 & 0.078 & 0.22 \\
\hline m400k-d100-s2 & $2.3 \times 10^{4}$ & 23273 & 4.2 & 0.057 & 0.16 \\
\hline $\mathrm{m} 400 \mathrm{k}-\mathrm{d} 100-\mathrm{s} 3$ & $4.3 \times 10^{4}$ & 42596 & 0.43 & 0.096 & 0.25 \\
\hline m400k-d10-s8 & $1.5 \times 10^{4}$ & 14978 & 1.4 & 0.037 & 0.38 \\
\hline m400k-d10-s9 & $2.8 \times 10^{4}$ & 27891 & 0.41 & 0.068 & 0.39 \\
\hline m10k-d100-s4 & $4.1 \times 10^{2}$ & 406 & 5.9 & 0.042 & 0.11 \\
\hline m10k-d100-s5 & $2.6 \times 10^{2}$ & 256 & 7.4 & 0.027 & 0.079 \\
\hline m10k-d100-s6 & $2.5 \times 10^{2}$ & 246 & 8.4 & 0.026 & 0.078 \\
\hline m10k-d10-s11 & 49 & 49 & $\cdots$ & 0.0049 & 0.00 \\
\hline m10k-d10-s12 & 61 & 61 & $\cdots$ & 0.0061 & 0.00 \\
\hline m10k-d10-s13 & 65 & 65 & $\ldots$ & 0.0065 & 0.00 \\
\hline
\end{tabular}

Notes. Here, "s" indicates the random seeds for the turbulence.

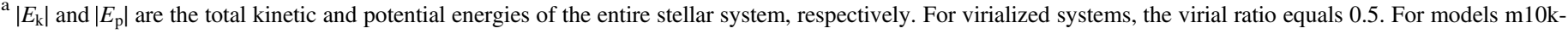
d10 we did not perform $N$-body simulations, and therefore their virial ratio is not calculated.

gas that is insufficiently dense to form stars tends to envelop the densest stellar conglomerates.

We now switch on the $N$-body code, for which we adopted the direct sixth-order Hermite predictor-corrector scheme (Nitadori \& Makino 2008) without gravitational softening and with an accuracy parameter of $\eta=0.1-0.25$. The total energy error over the time span of the $N$-body simulations remained below $\sim 10^{-3}$.

The sizes of the stars we adopted from the zero-age main sequence radii for solar metallicity stars (Hurley et al. 2000). We allow stars to collide using the sticky sphere approach. New stellar radii are assumed to be the zero-age main sequence radii for the new mass. Stellar mass loss was incorporated only at the end of the main sequence (Hurley et al. 2000; see Fujii et al. 2009; Fujii \& Portegies Zwart 2013, for the details).

We did not perform the $N$-body simulations for models m10k-d10 $\left(M_{\mathrm{g}}=10^{4} M_{\odot}\right.$ and $\left.\rho_{\mathrm{g}}=10 M_{\odot} \mathrm{pc}^{-3}=170 \mathrm{~cm}^{-3}\right)$ because the hydrodynamical simulations resulted in less than 100 stars, and we aim to have $\gtrsim 100 M_{\odot}$ star clusters. In these simulations even the densest regions were $<1000 M_{\odot} \mathrm{pc}^{-3}$.

\section{RESULTS}

\subsection{Formation of Embedded, Classical Open, and Young Massive Clusters}

The $N$-body simulations are started at what we will call $t=0$ Myr. The initial distribution of stars follows the distribution of the densest regions in the turbulent molecular cloud. In Figure 2 we present a time series of snapshots of model m400k-d100-s3. The entire system continuously expands because not all stars are bound after gas expulsion. The distribution of stars is clumpy, and it takes a few Myr before the stars assemble into a more coherent aggregate.

We interrupt the simulations twice, at $t=2$ and at $t=10 \mathrm{Myr}$, in order to analyze the stellar distribution and detect clustered aggregates. Clumps are found in these snapshots by means of HOP (Eisenstein \& Hut 1998) in AMUSE using an outer cutoff density of $\rho_{\text {out }}=4.5 M_{\mathrm{s}} /\left(4 \pi R_{\mathrm{h}}^{3}\right)$ (three times the half-mass density of the entire stellar system, $\rho_{\mathrm{h}}=M_{\mathrm{s}} /\left(8 \pi R_{\mathrm{h}}^{3}\right)$, where $M_{\mathrm{s}}$ is the total stellar mass and $R_{\mathrm{h}}$ is the half-mass-radius relation of the entire distribution of the stars), a saddle-point density threshold $\left(\rho_{\text {saddle }}=8 \rho_{\text {out }}\right)$, and the peak density threshold ( $\left.\rho_{\text {peak }}=10 \rho_{\text {out }}\right)$, and the number of particles for the neighbor search $\left(N_{\text {dense }}\right)$ and the number of particles to calculate the local density $\left(N_{\text {hop }}\right)$ are set to be 64 . The number of neighbors is used to determine which two groups merge, $N_{\text {merge }}=4$. With these settings, the detection limit of the clump mass is $\sim 100 M_{\odot}$. Sometimes HOP identifies multiple clumps as one, but by applying the method repeatedly we can separate those again. For this iterative procedure we adopt $\rho_{\text {out }}=\rho_{\mathrm{h}, \mathrm{c}}$, where $\rho_{\mathrm{h}, \mathrm{c}}$ is the half-mass density of a detected clump. We continue this procedure until $\rho_{\mathrm{h}, \mathrm{c}} \gtrsim 100 \rho_{\mathrm{h}}$, after which the clumps are so dense compared to the background that they do not separate anymore into substructures (see Fujii 2015b for the details).

In Figure 3 we present the mass and half-mass radius of the star clusters obtained from our simulations at $t=2$ and at $t=10$ Myr. For comparison, we added a number of observed open clusters (classical open, embedded, young massive, and leaky clusters) to the same diagram. The majority of the identified clusters have masses and radii consistent with those of classical open clusters (Piskunov et al. 2008) (see also Fujii 2015b) and of known embedded clusters (Lada \& Lada 2003). The densest initial molecular clouds (m1Md100, m400k-d100, and m400k-d10) tend to form massive compact clusters, similar to young massive clusters. Such compact clusters do not form in the less-dense or less-massive molecular clouds (such as m1M-d1 or m10k-d100).

When observing the 10-Myr-old stellar conglomerates from a distance, they tend to blend into a single star-forming region with an average density of $\sim 0.01 M_{\odot} \mathrm{pc}^{-3}$, which is comparable to the mean field density in the solar neighborhood 

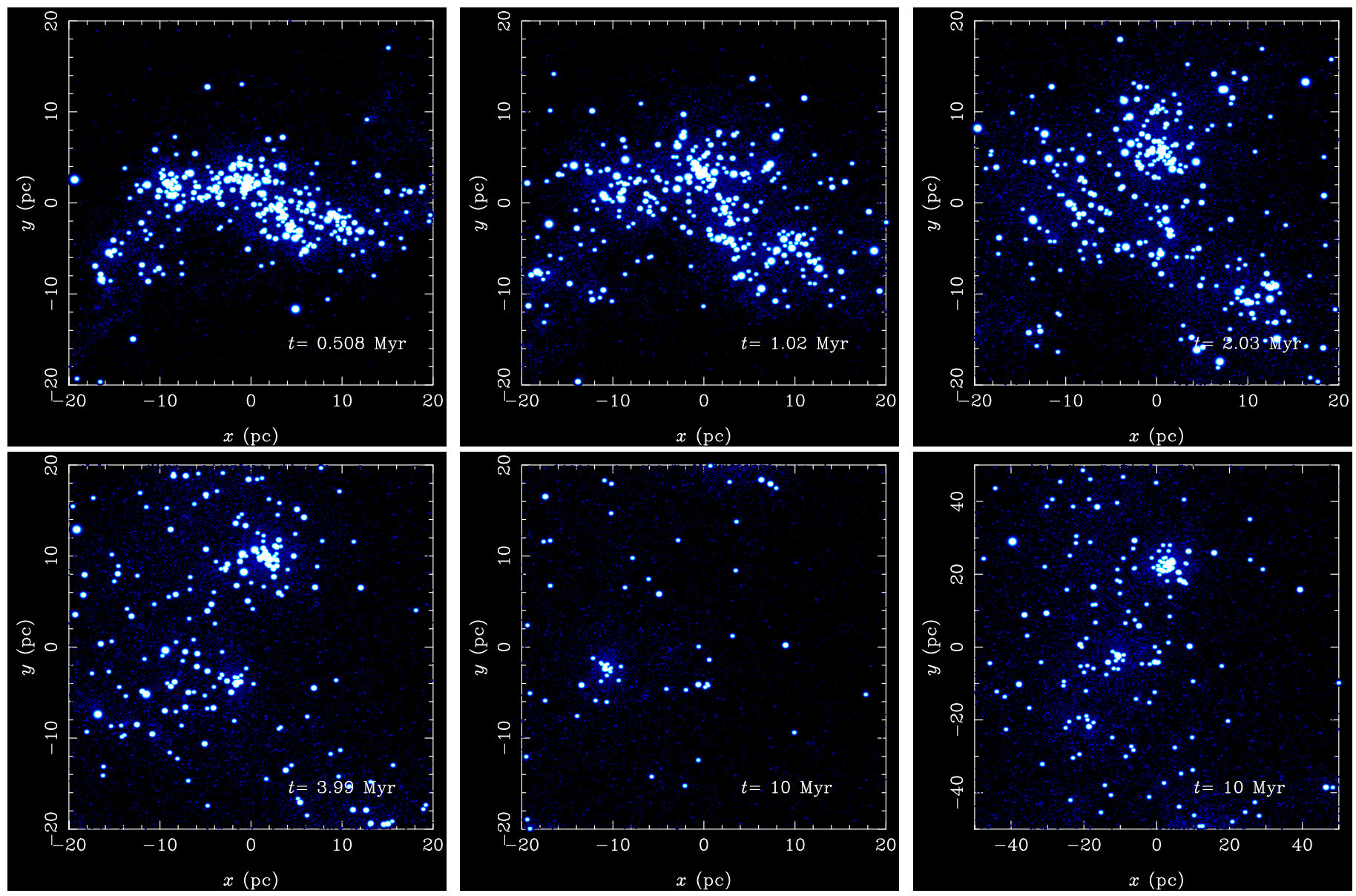

Figure 2. Snapshots of model m400k-d100-s3. The size of the white dots indicates the masses of the stars: $8<m / M_{\odot}<16$ for the small dots, $16<m / M_{\odot}<40$ for middle sized, and $m>40 M_{\odot}$ for the largest dots. Stars with a mass $m<8 M_{\odot}$ are plotted as small blue dots.
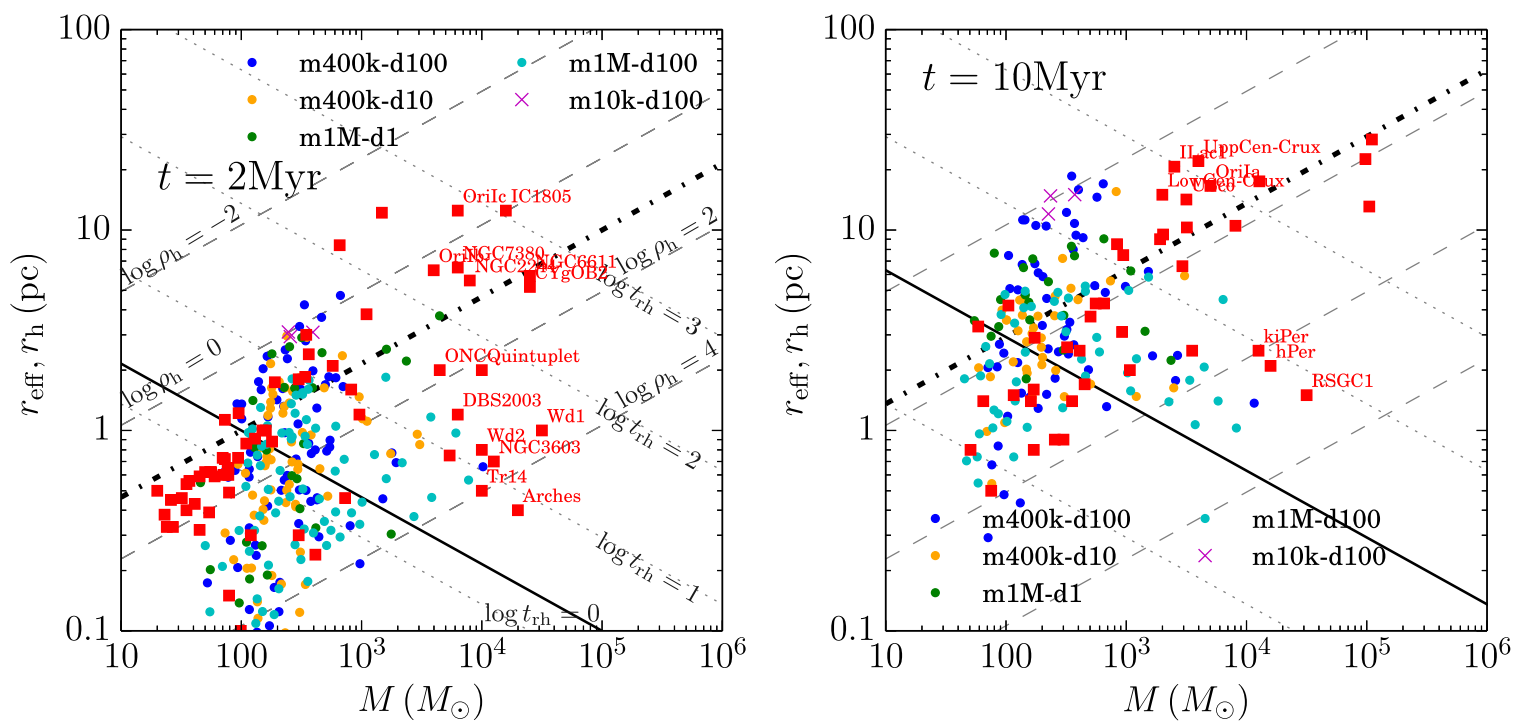

Figure 3. Mass-radius diagram of observed and simulated clusters at $t=2$ and $10 \mathrm{Myr}$ since the start of the $N$-body simulations. Colored dots are clusters obtained from simulations using a clump-finding method. Crosses indicate the median radius and the total mass of the entire stellar system rather than the detected individual clusters. Red squares indicate observed clusters with an age of 1-5 Myr (left) and 5-15 Myr (right). Data are from Piskunov et al. (2008), Winston et al. (2009), Luhman et al. (2003), Andersen et al. (2006), Fang et al. (2009), Levine et al. (2006), Flaherty \& Muzerolle (2008), Bonatto \& Bica (2011), Horner et al. (1997), Drew et al. (1997), Hodapp \& Rayner (1991), and Portegies Zwart et al. (2010). Observed clusters with names are the clusters listed in Pfalzner (2009) and Portegies Zwart et al. (2010). Black thick solid and dash-dotted lines indicate the lines at which the relaxation time and the dynamical time are equal to the age of the stellar populations. Gray dashed lines indicate the half-mass densities of $0.01,1,100$, and $10^{4} M_{\odot} \mathrm{pc}^{-3}$, and gray dotted lines indicate the half-mass relaxation times of 1000 , 100, 10, and $1 \mathrm{Myr}$ from top to bottom. We used the median radius for the observed leaky clusters (Wolff et al. 2007; Pfalzner 2009). 
(Holmberg \& Flynn 2000). Such conglomerates may remain unrecognizable as a cluster system. For those simulations in which no clumps are detected down to a limit of $100 M_{\odot}$, we adopt the median distance of the stars from the cluster center.

The masses and half-mass radii of the clusters in our simulations mainly resemble the populations of observed embedded and classical open clusters. This result appears to be independent of the initial molecular-cloud density. Embedded and classical open clusters cluster around the point where the cluster age $\left(t_{\text {age }}\right)$ equals the dynamical time $\left(t_{\text {dyn }}\right)$ and the half-mass relaxation time $\left(t_{\mathrm{rh}}\right)$ (see also Fujii 2015b).

Here the dynamical time and the half-mass relaxation time are written as

$$
t_{\mathrm{dyn}} \sim 2 \times 10^{4}\left(\frac{M}{10^{6} M_{\odot}}\right)^{-1 / 2}\left(\frac{r_{\mathrm{h}}}{1 \mathrm{pc}}\right)^{3 / 2} \text { year }
$$

and

$$
t_{\mathrm{rh}} \sim 2 \times 10^{8}\left(\frac{M}{10^{6} M_{\odot}}\right)^{1 / 2}\left(\frac{r_{\mathrm{h}}}{1 \mathrm{pc}}\right)^{3 / 2} \text { year }
$$

respectively (Portegies Zwart et al. 2010), where $M$ is the cluster mass, and $r_{\mathrm{h}}$ is the half-mass radius. For clarity we assumed that the virial radius of star clusters is comparable to the half-mass radius and that the mean stellar mass is $1 M_{\odot}$ (as is the case in our simulations). In Figure 3 we present lines on which the relaxation (black full) and dynamical (black dashdotted) times are equal to the age of the clusters, respectively. Both lines, as well as all the symbols, move upward with time.

For the formation of young massive clusters, we find that a dense, massive molecular cloud is necessary. The densities required to form such massive clusters exceed the density expected by Larson's relation; the velocity dispersion necessary for the formation of young massive clusters is too high. Such an initial high density may be realized by cloud-cloud collisions (Fukui et al. 2014). The velocity dispersion of our dense model is $\sim 20 \mathrm{~km} \mathrm{~s}^{-1}$, which is comparable to the typical relative velocity of molecular clouds associated with young massive clusters, such as NGC 3603 and Westerlund 2. For these clusters, a collision between two molecular clouds was considered to trigger their formation (Furukawa et al. 2009; Ohama et al. 2010; Fukui et al. 2013, 2014), which is consistent with our findings here.

To form a star cluster in our simulations, the molecular cloud must be compressive (a high velocity dispersion due to a high density), which is consistent with observations (Zinnecker \& Yorke 2007). From various initial conditions, we find that star clusters similar to open, known embedded, and young massive clusters form in these simulations, but leaky clusters $\left(M \sim 10^{4} M_{\odot}\right.$ and $\left.r_{\mathrm{h}} \sim 10 \mathrm{pc}\right)$ must form from different initial conditions. We discuss the formation of leaky clusters in the following section (Section 3.2).

\subsection{Formation of Leaky Clusters}

In the previous section, we show that known embedded, classical open, and young massive clusters form from turbulent molecular clouds, but no leaky cluster is found in our simulations. In this section we address the questions, How do leaky clusters form? Is the formation process different from the other clusters?
Pfalzner (2011) proposed that observed embedded clusters grow in mass and size due to star formation and become leaky clusters as a result of the expulsion of the residual gas. This scenario was later explored, and the evolutionary tracks of such a cluster on the mass-radius diagram were suggested (Parmentier \& Pfalzner 2013; Pfalzner \& Kaczmarek 2013; Pfalzner et al. 2014). Portegies Zwart et al. (2010), however, classified the leaky clusters as OB associations. Here we do not discuss if the leaky clusters are associations or clusters; we treat both leaky clusters and associations as less-dense clustered systems.

We consider leaky clusters (and also OB associations) to form clumpy distributions but that they lose this structure in the early dynamical evolution, contrary to the arguments in Pfalzner (2011). We support our argument with the simulation model m1M-d1-s16 (see the left panels in Figure 4). This simulation started with a spherical molecular cloud that collapsed asymmetrically because of the turbulent velocity field. Stars that formed mainly in the densest regions result in the stellar distribution being elongated and clumpy.

After the residual gas has been removed, the clusters tend to be supervirial, and some stars escape right away (see the virial ratio given in Table 2). As a consequence, the entire stellar distribution expands with time. At an age of $t=10 \mathrm{Myr}$ the density of the environment has decreased substantially, and the spatial distribution of the stars resembles leaky clusters and $\mathrm{OB}$ associations. In Figure 5 we present the spatial distribution of $\mathrm{O}$ and B spectral-type stars in the association Scorpius OB2 (Sco OB2), which can be compared with our simulations in Figure 4.

Sco OB2 is composed of three subgroups: Upper Scorpius (USco), Upper Centaurus-Lupus (Upper Cen-Lup), and Lower Centaurus-Crux (Lower Cen-Crux) (Wolff et al. 2007). These subgroups are listed in Pfalzner (2009) as leaky clusters and as associations in Portegies Zwart et al. (2010). They are all located at similar distances from the Sun, at $145 \mathrm{pc}, 142$, and $118 \mathrm{pc}$, respectively (Wolff et al. 2007), and therefore they are considered to be a system. The distribution of massive $(\mathrm{O}$ and B) stars in Sco OB2 is very similar to the distribution of massive stars in model m1M-d1-s16 at an age of $10 \mathrm{Myr}$.

In Figure 6 we present the result of our clump-finding analysis for model m1M-d1-s16 at $2 \mathrm{Myr}$ and at $10 \mathrm{Myr}$. At $t=2 \mathrm{Myr}$ we detected $\sim 20$ clusters that are similar to observed embedded star clusters. At $t=10 \mathrm{Myr}$ no clear massive clusters remain visible in the snapshot (see Figure 4), although we still detect several classic open cluster-like structures; in the epoch between 2 and $10 \mathrm{Myr}$, the stellar distribution has dispersed.

When interpreting the entire system in each simulation as a single association, the mass and radius are very similar to those of observed leaky clusters and OB associations. In Figure 6 we present these as crosses (near the top of the panels at 2 and $10 \mathrm{Myr}$ ). Model m1M-d1-s16 has an appearance and dynamical structure similar to the Sco OB2 system, rather than to the individual subclusters USco, Upper Cen-Lups, and Lower CenCrux. In this analysis we excluded single stars (those with a local density $\rho_{6}<10^{-3} M_{\odot} \mathrm{pc}^{-3}$ ), which is more than an order of magnitude lower than the mean density of the solar neighborhood ( $\rho_{6}$ here is the density measure within the six nearest neighbors).

We still detected clumps consistent with open clusters in model m1M-d1-s16. These clumps are the result of the clumpiness of molecular clouds at a time when we stop the 

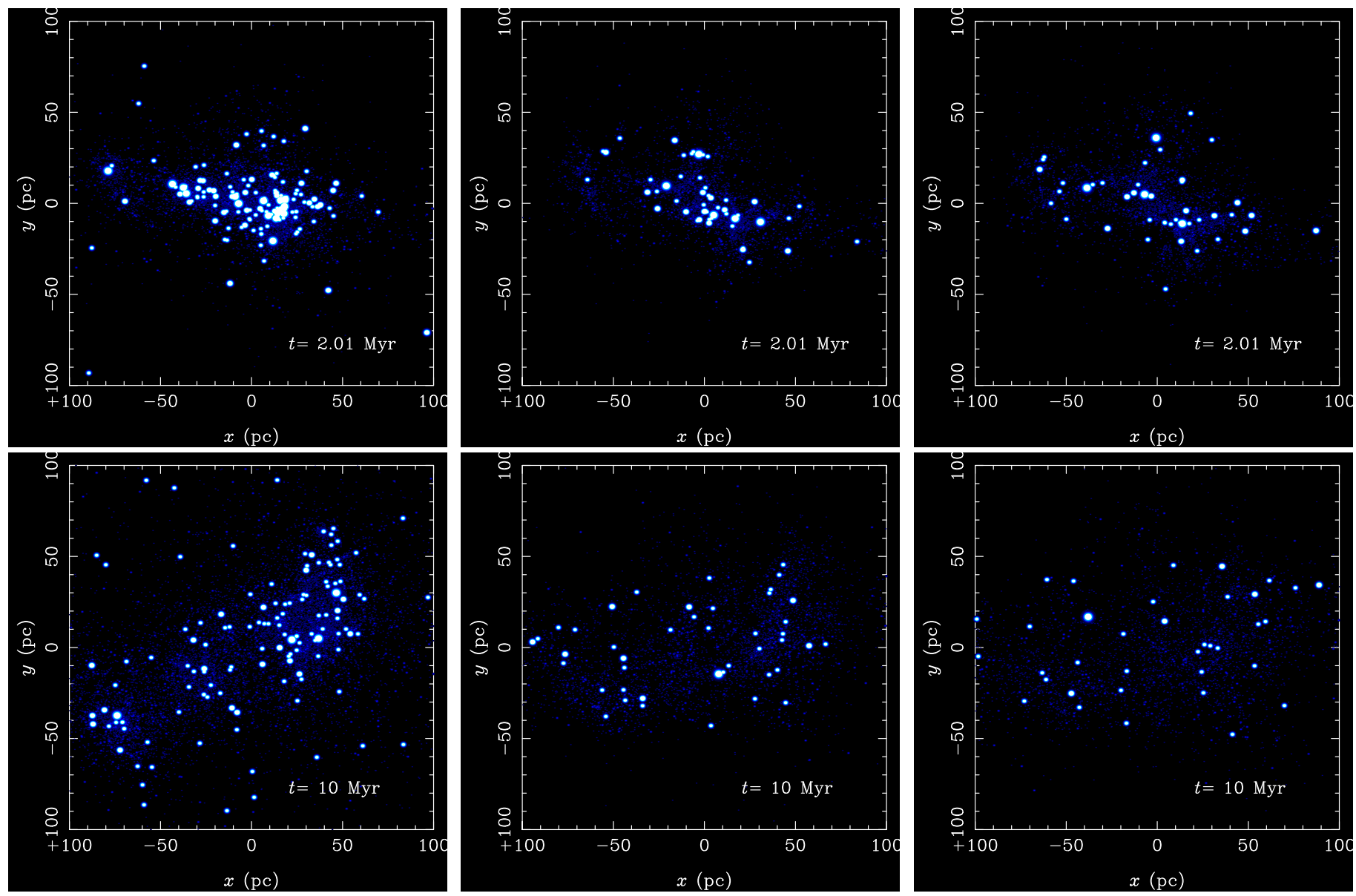

Figure 4. Snapshots at $t=2$ (top) and 10 (bottom) Myr for model d1-1M, but for different timing of gas removal: $t=0.9,0.75$, and $0.65 t_{\mathrm{ff}, \mathrm{i}}$ (models m1M-d1-s16, m1M-d1-s16-t0.75, and m1M-d1-s16-t0.65, respectively) from left to right.

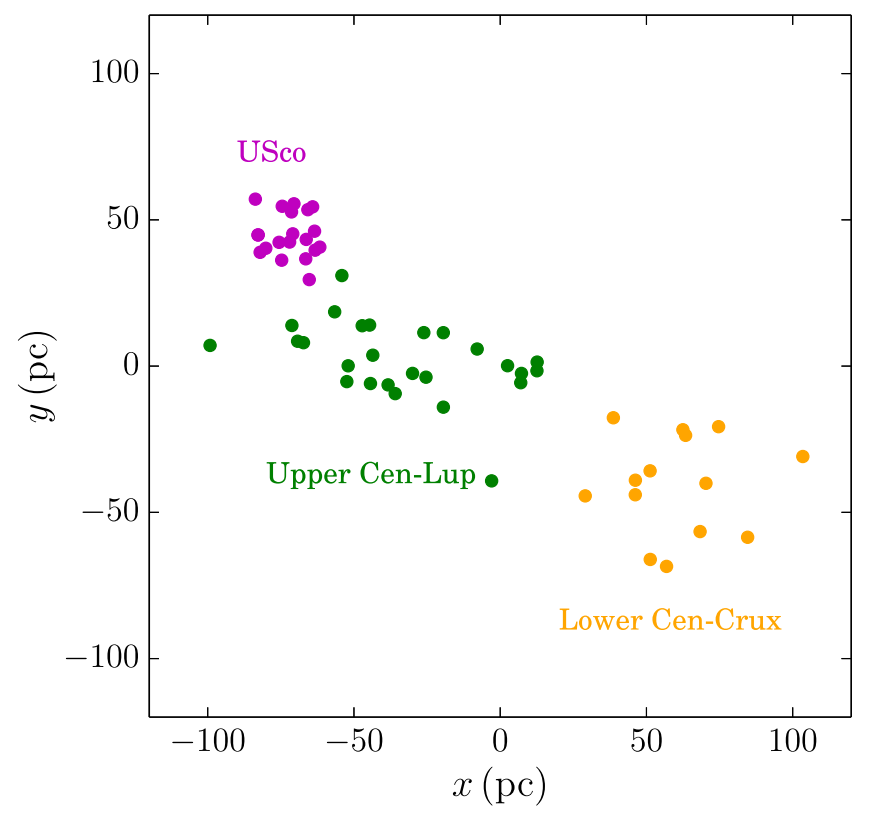

Figure 5. Positions of B-type stars that belong to USco (magenta), Upper CenLup (green), and Lower Cen-Crux (orange). Data are from Wolff et al. (2007).

We assume $140 \mathrm{pc}$ as the distance (Wolff et al. 2007). hydrodynamical simulations (at $\sim 0.9 t_{\mathrm{ff}, \mathrm{i}}$ ). In the observed starforming regions, however, stars appear to form when the local density exceeds some threshold density for self-gravitating clouds of $\sim 10^{3} \mathrm{~cm}^{-3}$ (McKee \& Ostriker 2007), and feedback starts to dominate the hydrodynamics as soon as the first massive star forms, which may happen well before a free-fall timescale. The free-fall timescale of model m1M-d1-s16 is $\sim 8 \mathrm{Myr}$, which is considerably longer than the formation time for massive stars ( 1 Myr) (McKee \& Ostriker 2007). In such a region, where the star-forming timescale is considerably smaller than the free-fall timescale of the entire molecular cloud, stellar feedback is expected to terminate the star formation before the molecular cloud fully collapses. This would result in a less-clumpy stellar distribution.

Unfortunately, in our simulations, we cannot take such gradual star formation and feedback processes into account, although they have been addressed with the AMUSE framework by Pelupessy \& Portegies Zwart (2012). In order to mimic the early star-formation process, we experimented with stopping the hydrodynamical simulations at an earlier epoch and replacing the gas particles with stellar particles.

As in our previous simulations, we assumed that the feedback terminates star formation and causes the residual gas to be ejected instantaneously. We stop the hydrodynamical 

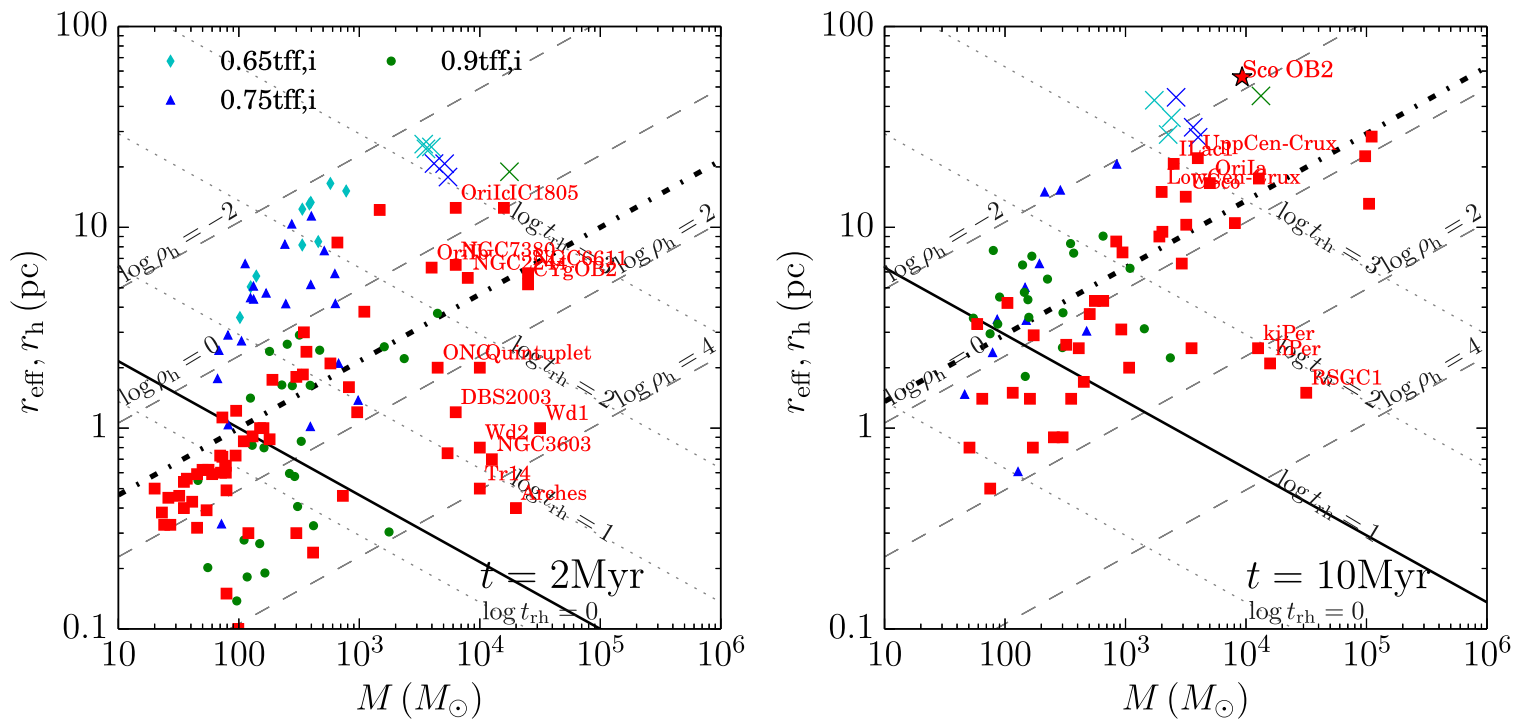

Figure 6. Mass-radius diagram of detected clusters for models m1M-d1-s16 (green dots), m1M-d1-s16-t0.75 (blue triangles), and m1M-d1-s16-t0.65 (cyan diamonds) at $t=2$ and 10 Myr. Red squares indicate observed young clusters; clusters with ages of 1-5 Myr and 5-15 Myr are plotted in the left and right panels, respectively. The data for the observed clusters are the same as those in Figure 3. The mass and half-mass radii of simulations, interpreted as unresolved clusters, are shown as crosses; each cross represents a single simulation.

simulation for model m1M-d1-s16 at $t=0.65 t_{\mathrm{ff}, \mathrm{i}}$ and $0.75 t_{\mathrm{ff}, \mathrm{i}}$ (5.3 and 6.2 Myr, respectively) and replace gas particles with stellar particles in the same way as for model m1M-d1-s16, i.e., assuming a local SFE given by Equation (2) and the same values for $\alpha_{\text {sfe }}=0.02$. The numbers of stars that form using this procedure decrease considerably, and the resulting virial ratio of the stellar system increases. We also run the same initial conditions but with different random seeds (m1M-d1-s15 and m1M-d1-s17). In Table 2 we present some global parameters for these models.

Snapshots of these models (m1M-d1-s16-t0.75 and m1Md1-s16-t0.65) are shown in the middle and right panels of Figure 4. The distribution of massive stars is less clumpy than that of model m1M-d1-s16 (standard model, in which the hydrodynamical simulation is stopped at $0.9 t_{\mathrm{ff}}$; see the left panels of Figure 4). We also apply the clump-finding algorithm to these models, the results of which are shown in Figure 6. At $t=2 \mathrm{Myr}$, several clumps are detected in both models, but they are less dense than those detected in our standard model. In model m1M-d1-s16-t0.65 in particular, the density of the detected clumps is only slightly elevated compared to the background density in the solar neighborhood $\left(0.01 M_{\odot} \mathrm{pc}^{-3}\right)$ (Holmberg \& Flynn 2000), and these clumps may therefore not be recognized as clusters. In model m1M-d1-s16-t0.75 some clumps that resemble open clusters are still detected at $t=10 \mathrm{Myr}$, but none in model m1M-d1-s16-t0.65. If we treat the entire system as one cluster, the masses are similar to those of leaky clusters and associations, even though the size remains larger by about a factor of two. In Figure 6 we present the mass and half-mass radius of the resulting clusters for stars with $\rho_{6}>10^{-3} M_{\odot} \mathrm{pc}^{-3}$.

Our assumption that star formation terminates instantaneously throughout the system after about one free-fall time of the molecular cloud probably overestimates the effect of the feedback considerably. In observed star-forming regions, the feedback from massive stars tends to limit star formation locally, but it may not affect the entire $(\sim 100 \mathrm{pc}$ across $)$ starforming region. In the simulations of Pelupessy \& Portegies
Zwart (2012), the wind of one massive $\sim 30 M_{\odot}$ star blows the residual gas from the clustered environment in a couple of Myr, which is much longer than that adopted in our simulations.

If star formation proceeds as clumpy as simulated here, the feedback is even more localized, which will result in a considerable age spread among subgroups. Our simulations would then be representative for the formation of cluster complexes such as USco, Upper Cen-Lups, and Lower CenCrux, or OB associations such as Sco OB2. The ages of these three subgroups are slightly different from each other: 14-15, 11-12, and 5-6 Myr for Upper Cen-Lup, Lower Cen-Crux, and USco, respectively (Wolff et al. 2007). If we could assume local feedback processes, an association (or leaky clusters) similar to Sco OB2 might form from an initial condition, such as models m1M-d1. Less-dense clusters tend to have wider age spreads (Parmentier et al. 2014), which is also consistent with our simulations. We therefore argue that the ancestors of associations are conglomerates of denser embedded clusters. We detect these as an environment with multiple low-mass but rather dense clusters that disperse in time. The evaporation of these clusters is driven by relaxation and feedback, and this makes them resemble associations.

\section{INITIAL CONDITIONS OF MOLECULAR CLOUDS}

In the previous section, we showed that our dense models tend to form young massive clusters and that less-dense models lead to leaky clusters as well as known embedded and classical open clusters. The type of the resulting star clusters is sensitive to the initial conditions of the parental molecular clouds. In this section, we compare our initial conditions with observed molecular clouds and discuss a model for the formation of clusters in the Milky Way and other nearby galaxies.

In Figure 7, we present the mass and density of individual molecular clouds observed in the Milky Way and those estimated for local disk and starburst galaxies (Krumholz et al. 2012). We also show the initial conditions of our simulations. The dashed line in Figure 7 indicates the Larson's 


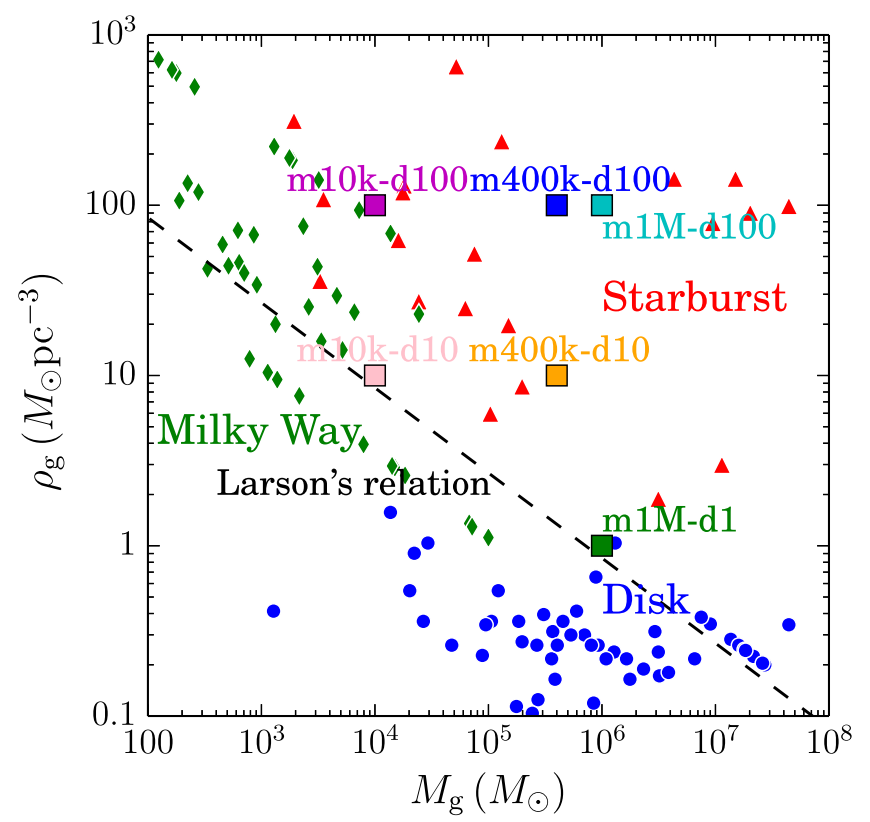

Figure 7. Mass-density relation of observed molecular clouds. Green diamonds indicate individual molecular clouds in the Milky Way galaxy. Blue circles and red triangles are for molecular clouds typical in individual local disk and starburst galaxies, respectively. Each point indicates one galaxy. The data are from Krumholz et al. (2012). Color squares indicate our initial conditions, which are the same as those shown in Figure 1. The dashed line indicates the mass-density relation following Larson's relation for a virialized cloud $\left(\sigma_{\mathrm{g}}^{2}=G M_{\mathrm{g}} / R_{\mathrm{g}}\right)$.

relation. In order to estimate the mass of molecular clouds following Larson's relation, we assume that the molecular clouds are in virial equilibrium (i.e., they satisfy $\sigma_{\mathrm{g}}^{2}=G M_{\mathrm{g}} / r_{\mathrm{g}}$, where $\sigma_{\mathrm{g}}, M_{\mathrm{g}}$, and $r_{\mathrm{g}}$ are the velocity dispersion, mass, and radius of the molecular clouds, respectively). Observed molecular clouds, however, are not necessarily virialized.

Molecular clouds in the Milky Way tend to follow Larson's relation, but with a large scatter of the density. On the other hand, not all of our initial conditions are consistent with the mass and density of molecular clouds observed in the Milky Way. Models m10k-d100 $\left(10^{4} M_{\odot}\right.$ and $100 M_{\odot} \mathrm{pc}^{-3} \simeq$ $\left.1700 \mathrm{~cm}^{-3}\right)$ and $\mathrm{m} 10 \mathrm{k}-\mathrm{d} 10 \quad\left(10^{4} M_{\odot}\right.$ and $10 M_{\odot} \mathrm{pc}^{-3} \simeq$ $170 \mathrm{~cm}^{-3}$ ), for example, are initially indistinguishable from typical molecular clouds in the Milky Way.

As we described in Section 3, the number of stars formed in model m10k-d10 was too small (fewer than 100 stars) to be recognized as a cluster in our analysis. Model m10k-d100 produces a sufficiently large number of stars but does not form a recognizable cluster after $2 \mathrm{Myr}$. If we treat the entire region of this model as a cluster conglomerate, the mass and radius are similar to that of an open cluster. From this, we conclude that the molecular clouds typical in the Milky Way tend to form classical open clusters, but that they are insufficiently massive and dense to form massive star clusters.

Model m1M-d1 $\left(10^{6} M_{\odot}\right.$ and $\left.1 M_{\odot} \mathrm{pc}^{-3}\right)$ represents the most massive molecular cloud in the Milky Way (Murray 2011), and it follows Larson's relation. This initial condition results in several embedded cluster cores, which eventually evolve to a conglomerate of associations.

The initial conditions that tend to form young massive clusters are considerably denser than the molecular clouds observed in the Milky Way (see Figure 7). To form young massive clusters in our simulations, a mass of at least several
$10^{5} M_{\odot}$ and a mean density of $10 M_{\odot} \mathrm{pc}^{-3}\left(170 \mathrm{~cm}^{-3}\right)$ are required. Such initial conditions are common in local starburst galaxies, but very rare in the Milky Way.

In Figure 7 we present the estimated mass and density of molecular cloud density typical for local starburst and disk galaxies. These data are obtained from Krumholz et al. (2012). We calculated the masses and densities for these molecular clouds from the free-fall timescale provided by Krumholz et al. (2012) using the observed surface gas densities $\left(\Sigma_{\mathrm{g}}\right)$. Krumholz et al. (2012) considered two rather distinct regimes of molecular clouds: the molecular cloud regime and the Toomre regime. The molecular cloud regime is expected to be common in local disk galaxies. The molecular clouds are decoupled from their surrounding interstellar medium and as a result are self-gravitating (Krumholz et al. 2012). The Toomre regime is common in starburst galaxies. In this case the interstellar medium is highly turbulent, and therefore the free-fall timescale of the molecular clouds should be estimated using the midplane pressure in the galactic disks (see Krumholz et al. 2012, for the details).

Following the description of Krumholz et al. (2012), we estimate the typical mass of molecular clouds for each galaxy listed in Krumholz et al. (2012). We take the smaller free-fall timescale for the molecular cloud and Toomre regimes $\left(t_{\mathrm{ff}, \mathrm{GMC}}\right.$ and $t_{\mathrm{ff}, \mathrm{T}}$, respectively) as the free-fall timescale $\left(t_{\mathrm{ff}}\right)$, which is consistent with Krumholz et al. (2012). We calculate the density through the free-fall timescale using

$$
\rho_{\mathrm{g}}=\frac{3 \pi}{32 G t_{\mathrm{ff}}^{2}} .
$$

In the Toomre regime, the midplane pressure in the disk of surface gas density $\Sigma_{\mathrm{g}}$ is

$$
P=\rho_{\mathrm{g}, \mathrm{T}} \sigma_{\mathrm{g}}^{2}=\phi_{P} \frac{\pi}{2} G \Sigma_{\mathrm{g}}^{2} .
$$

Here $\rho_{\mathrm{g}, \mathrm{T}}$ is the molecular cloud density in the Toomre regime, $\sigma_{\mathrm{g}}$ is the velocity dispersion of the gas, and $\phi_{P}$ is a dimensionless factor (Krumholz et al. 2012). The Toomre $Q$ for the gas is written as

$$
Q=\frac{\sqrt{2(\beta+1)} \sigma_{\mathrm{g}} \Omega}{\pi G \Sigma_{\mathrm{g}}} .
$$

Here $\beta$ is the logarithmic index of the rotation curve $(\beta=0$ for a flat rotation curve, whereas for solid-body rotation $\beta=1$ ), and $\Omega=2 \pi / t_{\text {orb }}$ ( $t_{\text {orb }}$ is the galactic orbital period) is the angular velocity of galactic rotation (see also Krumholz \& McKee 2005). From these equations, the density of the molecular cloud becomes

$$
\rho_{\mathrm{g}, \mathrm{T}} \simeq \frac{(\beta+1) \phi_{P} \Omega^{2}}{\pi G Q^{2}} .
$$

Here we adopt $Q \sim 1$ and $\beta=0$ following Krumholz et al. (2012). If we assume that the cloud is virialized-i.e., $\sigma_{\mathrm{g}}^{2} \sim G M_{\mathrm{g}, \mathrm{T}} / r_{\mathrm{g}}$, where $M_{\mathrm{g}, \mathrm{T}}$ and $r_{\mathrm{g}}$ are the mass and radius of the cloud-from Equation (7) and $\rho_{\mathrm{g}, \mathrm{T}}=3 M_{\mathrm{g}, \mathrm{T}} /\left(4 \pi r_{\mathrm{g}}^{3}\right)$, we can estimate the cloud mass using

$$
M_{\mathrm{g}, \mathrm{T}} \sim \frac{1}{32} \sqrt{\frac{3}{\pi}} G^{3 / 2} \Sigma_{\mathrm{g}}^{3} t_{\mathrm{orb}}^{3} \rho_{\mathrm{g}}^{-1 / 2} .
$$


Because for each galaxy $t_{\text {orb }}$ and $\Sigma_{\mathrm{g}}$ are given in Krumholz et al. (2012), we can estimate the cloud density and mass from Equations (8) and (9). Here we adopt $\phi_{P} \simeq 3$, following Krumholz et al. (2012).

For the molecular cloud regime (i.e., $t_{\mathrm{ff}, \mathrm{GMC}}<t_{\mathrm{ff}, \mathrm{T}}$ ), the mass is estimated as follows. The mass of molecular clouds is estimated by the two-dimensional Jeans mass in galactic disks (Kim \& Ostriker 2002; McKee \& Ostriker 2007; Chandar et al. 2011), which is given by

$$
M_{\mathrm{g}, \mathrm{GMC}}=\frac{\sigma_{\mathrm{g}}^{4}}{G^{2} \Sigma_{\mathrm{g}}},
$$

(see Equation (3) of Krumholz et al. 2012). Since the mass and density of molecular clouds in the molecular cloud regime are written as $M_{\mathrm{g}, \mathrm{GMC}}=\pi r_{\mathrm{g}}^{2} \quad \Sigma_{\mathrm{GMC}}$ and $\rho_{\mathrm{g}, \mathrm{GMC}}=$ $(3 / 4 \pi) M_{\mathrm{g}, \mathrm{GMC}} r_{\mathrm{g}}^{-3}$, where $\Sigma_{\mathrm{GMC}}$ is the surface density of molecular clouds, using Equation (10) we can calculate the density of molecular clouds with (Equation (4) in Krumholz et al. 2012)

$$
\rho_{\mathrm{g}, \mathrm{GMC}}=\frac{3 \sqrt{\pi}}{4} \frac{G \sqrt{\sum_{\mathrm{GMC}}^{3} \Sigma_{\mathrm{g}}}}{\sigma_{\mathrm{g}}^{2}} .
$$

Here we adopt $\Sigma_{\mathrm{GMC}}=85 \mathrm{M}_{\odot} \mathrm{pc}^{-2}$ and $\sigma_{\mathrm{g}}=8 \mathrm{~km} \mathrm{~s}^{-1}$ for all galaxies following Krumholz et al. (2012). Using Equations (10) to (11), we obtain the mass and density in the molecular cloud regime using the value for $\Sigma_{\mathrm{g}}$ from Krumholz et al. (2012).

The obtained masses and densities for molecular clouds in the local disk and starburst galaxies are presented in Figure 7. Most galaxies are in the Toomre regime (and only 13 disk galaxies are in the molecular cloud regime). The molecular clouds typical for starburst galaxies are factors of 10-100 denser than those following Larson's relation. Our massive and dense models (m400k-d100, m400k-d10, and m1M-d100), which form young massive clusters, are consistent with the molecular cloud observed in starburst galaxies. Starburst galaxies such as M83 (Bastian et al. 2011) and M51 (Chandar et al. 2011) are indeed rich in dense, massive clusters. On the other hand, molecular clouds typical in local disk galaxies do follow Larson's relation. Our model m1M-d1, which forms classical open and leaky clusters (associations), appears to be quite similar to these molecular clouds.

The typical molecular cloud in a disk galaxy, such as the Milky Way, tends to form classical open clusters and associations, but these clouds are insufficiently massive to form young massive star clusters. This is consistent with the abundance of open star clusters and associations in the Milky Way and with the lack of massive star clusters. According to our simulations, the formation of a massive star cluster requires a massive $\left(\sim 10^{5}-10^{6} M_{\odot}\right)$ and dense $\left(\sim 10-100 M_{\odot} \mathrm{pc}^{-3}\right)$ molecular cloud. Such a massive molecular cloud has, if virialized, a velocity dispersion of $\sim 20 \mathrm{~km} \mathrm{~s}^{-1}$. Such a high velocity dispersion (under compressive conditions) could result from the collision between two clouds (Furukawa et al. 2009; Ohama et al. 2010; Fukui et al. 2014). Comparable high velocities are observed in the regions surrounding young massive clusters, such as in the vicinity of NGC 3603 (Fukui et al. 2014) and Westerlund 2 (Furukawa et al. 2009; Ohama et al. 2010). These clusters are claimed to have been the result of cloud-cloud collisions (Furukawa et al. 2009; Ohama et al. 2010; Fukui et al. 2014). These claims are supported by three-dimensional magnetohydrodynamic simulations, which also suggest that such cloud-cloud collisions initiate the formation of massive cloud cores and potentially form massive star clusters (Inoue \& Fukui 2013).

Although our initial conditions of molecular clouds cover a relatively wide range of mass and density, they are limited by our choice to opt for homogeneous-density spheres. Recent numerical studies indicate that molecular clouds with a concentrated density profile such as a power law tend to form one high-mass star in the center surrounded by many low-mass stars (Girichidis et al. 2011, 2012). Such centrally concentrated models then may more efficiently lead to the formation of massive clusters than our adopted homogeneous initial conditions.

\section{MASS AND RADIUS EVOLUTION OF YOUNG STAR CLUSTERS}

Star clusters can be subdivided into several types, which represent themselves clearly when presented in a mass-radius diagram. The mass-radius distribution of star clusters changes with time. Here we discuss the time evolution of the mass and radius of young clusters.

\subsection{Observations}

We start with summarizing the mass and radius evolution of observed young star clusters. These observations are presented in Figure 8, in particular for observed embedded clusters, classical open star clusters, young massive (starburst) clusters, and associations (Hodapp \& Rayner 1991; Drew et al. 1997; Horner et al. 1997; Lada \& Lada 2003; Luhman et al. 2003; Andersen et al. 2006; Levine et al. 2006; Flaherty \& Muzerolle 2008; Piskunov et al. 2008; Fang et al. 2009; Pfalzner 2009; Winston et al. 2009; Portegies Zwart et al. 2010; Bonatto \& Bica 2011). For clarity we bin the clusters in age in intervals of $t_{\mathrm{age}}=1-5 \mathrm{Myr}, 5-20 \mathrm{Myr}$, and 20-100 Myr.

Pfalzner (2009) and Portegies Zwart et al. (2010) list several young massive clusters, but in many cases the listed radii differ. We adopt the half-mass radius given in Portegies Zwart et al. (2010) because the radius presented in Pfalzner (2009) corresponds to the core radius of the clusters rather than the half-mass radius. The former gives a more direct comparison with our simulations. In our analysis we try to stay as much as possible to the same definition of cluster radius. Piskunov et al. (2008) present projected core and tidal radii by fitting King models (King 1966). Because the density profiles for the open clusters listed in Piskunov et al. (2008) are very shallow, we adopted their core radii, which for a King model with $W_{0}=3$ is quite similar to the half-mass radius (the ratio of the threedimensional core radius to half-mass radius is 0.65 for a King model with $W_{0}=3$ ).

Embedded clusters observed in the Milky Way galaxy reside almost exclusively in the left panel of the mass-radius diagram ( $t=1-5 \mathrm{Myr}$ panel in Figure 8 ) because they are young by definition. Embedded and classical open clusters populate the same region (at the bottom left in the same panel). These clusters tend to grow in size with age, which is a consequence of relaxation and outgassing; embedded clusters observed in the Milky Way therefore appear as ancestors of classical open clusters (Fujii 2015b). Associations populate the top right 

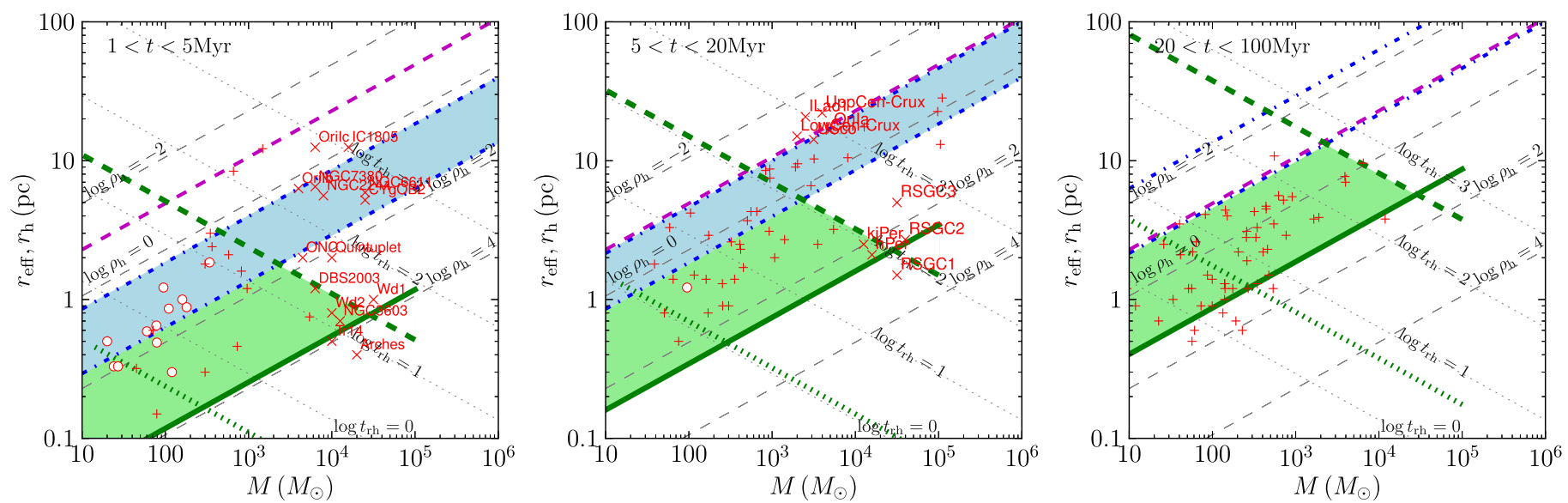

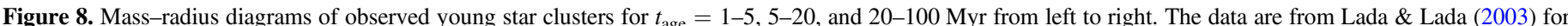

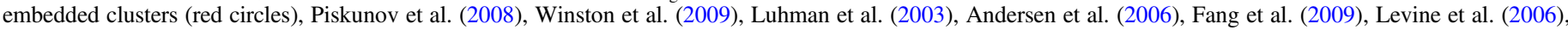

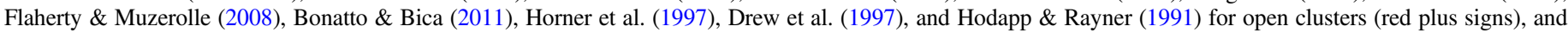

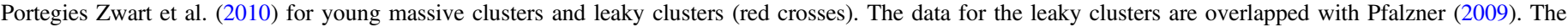
clusters listed in Portegies Zwart et al. (2010) are shown with the names.

region of the left and middle panels $(t=1-5 \mathrm{Myr}$ and 5-20 Myr, respectively), and young massive clusters are found to the right in the diagrams in Figure 8. As was already suggested by Pfalzner (2009), young massive star clusters are well separated in mass and radius from embedded and open clusters. This separation, however, diminishes for the older age group (20-100 Myr; see the right panel of Figure 8).

\subsection{Analytical Model for the Dynamical Evolution of Young Star Clusters}

The distribution and evolution of the observed star clusters in mass and radius can be understood from our models of the dynamical evolution for star clusters.

The lower limit of the cluster density can be understood by considering the background density in the field. The magenta dashed line in the diagram indicates $\rho=0.1 M_{\odot} \mathrm{pc}^{-3}$, which is an order of magnitude higher than the mean density of the field stars in the solar neighborhood (Holmberg \& Flynn 2000). We adopt $\rho=0.1 M_{\odot} \mathrm{pc}^{-3}$ as a lower limit for the cluster density (magenta dashed line in Figure 8). Star clusters with a density similar to or lower than the mean stellar density would therefore not be recognizable as clusters. And indeed, only a few of the most massive clusters reside above this curve, and those have a relatively high concentration. As a consequence, their core densities exceed the local density considerably, which helps to identify them as clusters in observational campaigns.

The blue dash-dotted lines in Figure 8 indicate the massradius relation for which the dynamical timescale (see Equation (3)) is equal to the age of the cluster. Each panel contains two lines, one for the minimum and one for the maximum age of the clusters shown in the panels. The region between these lines is shaded blue. Clusters between or below the blue lines will be recognizable as bound systems unless the lines exceed the magenta dashed line. Portegies Zwart et al. (2010) and Gieles \& Portegies Zwart (2011) argued that the ratio between cluster age and dynamical time provides a good indicator for separating the bound from the unbound systems: they adopt as a criterion $t_{\mathrm{age}} / t_{\mathrm{dyn}} \gtrsim 3$ to make this distinction. Using this criterion, they categorized the leaky clusters in Pfalzner (2009) as associations. The blue region in Figure 8 moves upward with time, together with the observed clusters. At $t>20 \mathrm{Myr}$ (the right panel in Figure 8) the blue lines are located above the magenta line, indicating that these clusters have a density too low to be recognized as clusters.

The evolution of dense star clusters is quite different from those of open clusters or associations. Dense star cluster evolution can roughly be divided into two phases: before core collapse and after core collapse. In the former phase, the core radius of the star clusters shrinks, and as a consequence its core density increases (Hénon 1965; Lynden-Bell \& Wood 1968). From the moment the first hard binaries form in the cluster core (Spitzer \& Hart 1971; Aarseth 1974), they act as energy sources (Heggie 1975; Hut 1983), causing the core to reexpand. From this moment on, the core- and half-mass radii of clusters increases. These processes proceed on the half-mass relaxation time:

$$
t_{\mathrm{rh}}=\frac{0.065 \sigma^{3}}{G^{2}\langle m\rangle \rho \ln \Lambda} .
$$

Here $\sigma$ and $\rho$ are the velocity dispersion and density of the cluster, respectively, and $\ln \Lambda$ is the Coulomb logarithm (Spitzer 1987). We rewrite Equation (12) to include some common dimensions in Equation (4).

Gieles et al. (2011) modeled the postcollapse evolution of the half-mass radius and density of star clusters due to the energy flux from the core, following the description of Hénon (1965). We attempt to understand the dynamical evolution of young star clusters using their description. We ignore the precollapse phase and consider only the evolution in the postcollapse (expansion) phase because the precollapse phase is much shorter than the postcollapse. The core-collapse time, which is the time for the precollapse phase, scales with the relaxation time (see Equation (4) or (12)). This timescale depends on the stellar mass function, and for clusters with a realistic mass function the core-collapse time is generally shorter than one relaxation (Portegies Zwart \& McMillan 2002; Gürkan et al. 2004; Fujii \& Portegies Zwart 2014). Since most of the young open clusters in our observed sample have a relaxation time $\lesssim 10 \mathrm{Myr}$ (see the left panel of Figure 8 ), they probably reach core collapse well within a few Myr. We also ignore the effect of the Galactic tidal field because the timescale 
we treat here is short $(<100 \mathrm{Myr})$ compared to the timescale for the tidal disruption ( $1 \mathrm{Gyr})$ (Gieles et al. 2007).

The time of the half-mass radius of clusters due to binary heating in the core is given by Equation (B7) in Gieles et al. (2011):

$$
r_{\mathrm{h}} \simeq\left(\frac{3 G}{4 \pi N}\right)^{1 / 3}(125 \zeta t)^{2 / 3} .
$$

Here $N$ is the initial number of stars in the cluster. In Equation (B7) in Gieles et al. (2011), the cluster mass is assumed as $M=\langle m\rangle N$, where $\langle m\rangle$ is the mean stellar mass. They adopted a scaled mass of $\langle m\rangle=0.5$, and as a result their Equation (B7) is slightly different from our equation. If we assume that $\langle m\rangle=0.5 M_{\odot}$, we can write this equation as

$$
r_{\mathrm{h}} \simeq 2.0 \zeta^{2 / 3}\left(\frac{M}{M_{\odot}}\right)^{-1 / 3}\left(\frac{t_{\mathrm{age}}}{\mathrm{Myr}}\right)^{2 / 3} \mathrm{pc} .
$$

Here we adopt $t=t_{\text {age }}$ because we ignore the precollapse phase. The expansion-rate coefficient, $\zeta$, depends on the ratio of the maximum to the minimum mass in the stellar mass function, $\mu \equiv m_{\max } / m_{\min }$. In Gieles et al. (2011) we use $\zeta \simeq 0.2$, which corresponds to $\mu \simeq 10$, and which is appropriate for globular clusters. Young clusters, however, should have a larger value of $\mu$ because of the presence of massive stars. For some of these clusters, $m_{\max } / m_{\min } \simeq 100 M_{\odot} /$ $0.01 M_{\odot} \simeq 10^{4}$. Following Gieles et al. (2011) and assuming $\zeta \propto \mu^{1 / 2}$, we obtain $\zeta \simeq 20$ for $\mu \simeq 10^{4}$. Equation (14) with $\zeta=20$ for $t=t_{\text {age }}=2,10,40 \mathrm{Myr}$ is shown as green dashed lines in Figure 8.

The majority of the observed clusters are located below this evolutionary line rather than straddling the line, which indicates that they have $\zeta<20$. The green dotted lines in each panel of Figure 8 show Equation (14) with $\zeta=0.2$. Most of the observed embedded and classical open clusters are located between the dotted green (for $\zeta=0.2$ ) and the dashed green $(\zeta=20)$ lines. This may be caused by the large dispersion in $\zeta$, as we discussed here, or because the precollapse time is not taken into account in our analysis. By ignoring the precollapse time we reduce the evolution time of a star cluster compared to the expectation.

The descriptions of Gieles et al. (2011) (Equations (13) and (14)) give infinite density at $t=0 \mathrm{Myr}$, which hardly seems realistic for actual young star clusters. Instead, we adopt Equation (B4) of Gieles et al. (2011):

$$
\rho_{\mathrm{h}} \simeq \frac{1}{G}\left(\frac{N}{250 \zeta t}\right)^{2}
$$

which gives the half-mass density as a function of time. We also adopt $\langle m\rangle=0.5 M_{\odot}$. We assume a (maximum) half-mass density of $10^{4} M_{\odot} \mathrm{pc}^{-3}$ at $t_{\text {age }}=0.1 \mathrm{Myr}$ irrespective of the cluster mass; we obtain $\rho_{\mathrm{h}}\left(M_{\odot} \mathrm{pc}^{-3}\right)=100(t / \mathrm{Myr})^{-2}$. Since $\rho_{\mathrm{h}}=3 M /\left(8 \pi r_{\mathrm{h}}^{3}\right)$, the relation can be written as

$$
r_{\mathrm{h}}=0.0158\left(\frac{M}{M_{\odot}}\right)^{1 / 3}\left(\frac{t_{\mathrm{age}}}{\mathrm{Myr}}\right)^{2 / 3}(\mathrm{pc}) .
$$

Equation (16) is presented in Figure 8 as the solid green line. One naively expects that clusters with an initial density smaller than $10^{4} M_{\odot} \mathrm{pc}^{-3}$ populate the area above this line, which is

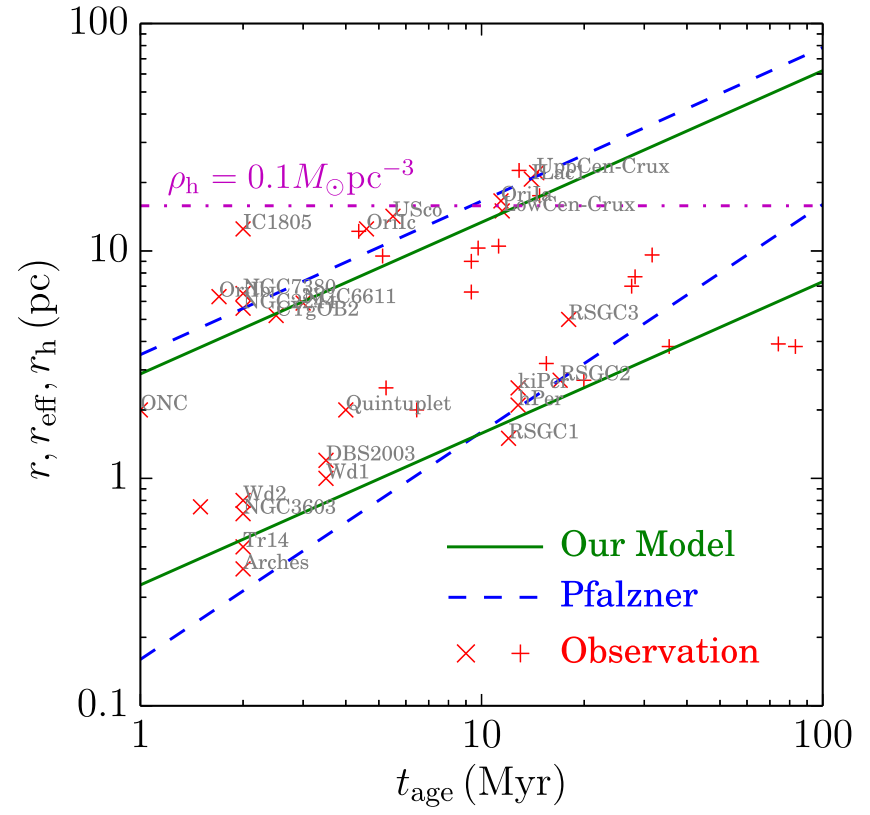

Figure 9. Cluster radius as a function of time for clusters with a mass of $10^{3}$ $10^{5} M_{\odot}$. Red cross signs are from Portegies Zwart et al. (2010) and red pluses from the others (see the caption of Figure 8). Blue dashed lines are the relations given in Pfalzner $(2009,2011): r=3.5 t_{\text {age }}^{2 / 3}$ and $r=0.16 t_{\text {age }}$ for top and bottom, respectively. Green lines are cluster radii as a function of time obtained from our model: $r_{\mathrm{h}}=2.7 t_{\text {age }}^{2 / 3}$ and $r_{\mathrm{h}}=0.34 t_{\text {age }}^{2 / 3}$ for top and bottom, respectively. Note that for starburst clusters we plot the half-mass radii given in Portegies Zwart et al. (2010) instead of "size" in Pfalzner (2009).

consistent with the observations. From a theoretical perspective we argue that star clusters are expected to reside in the green and blue regions in Figure 8, which for the majority of observed clusters appears to be the case.

From these results, the regions in which clusters are expected to exist on mass-radius diagrams are shown by the green and blue shades in Figure 8, and the observed distribution of star clusters matches them. Furthermore, our analytical models suggest two distinct populations of massive $\left(\sim 10^{4} M_{\odot}\right)$ clusters, which are called starbursts and leaky clusters by Pfalzner (2009). We argue that these two populations naturally appear if we consider the formation and the dynamical evolution process of star clusters.

\subsection{Time Evolution of Cluster Radius: Leaky and Starburst Clusters}

Young star clusters with $M \sim 10^{4} M_{\odot}$ are divided into two groups, as can be seen in Figure 8. Pfalzner (2009) named them starburst (young massive) clusters and leaky clusters (following Portegies Zwart et al. 2010 we identify the latter category as associations). Pfalzner $(2009,2011)$ showed that both families of clusters expand with time, but at a different rate: $r / \mathrm{pc}=0.16\left(t_{\mathrm{age}} / \mathrm{Myr}\right)$ for the starburst clusters and $r / \mathrm{pc}=3.5\left(t_{\mathrm{age}} / \mathrm{Myr}\right)^{2 / 3}$ for the associations. In this section, we discuss the origin of these different evolutionary tracks.

In Figure 9 we present the age and radius of observed young star clusters with a mass of $10^{3}<M<10^{5} M_{\odot}$. The time evolution for cluster radii, plotted as the solid green lines, is obtained from the analytic models discussed in Section 5.2.

Associations are about one dynamical timescale old, and therefore we can hardly confirm whether they are bound or not. If we consider them to be one dynamical timescale old, i.e., 
$t_{\text {age }} \simeq t_{\text {dyn }}$, Equation (3) gives $r_{\mathrm{h}} / \mathrm{pc}=2.7\left(t_{\mathrm{age}} / \mathrm{pc}\right)^{2 / 3} . \mathrm{We}$ present this relation in Figure 9 as the top green line. The model is consistent with the observed clusters, and the power-law index of our model is consistent with that of Pfalzner (2011).

For starburst clusters, we adopt the results based on Gieles et al. (2011). By adopting $M=10^{4} M_{\odot}$ in Equation (16), we obtain $r_{\mathrm{h}} / \mathrm{pc}=0.34\left(t_{\mathrm{age}} / \mathrm{Myr}\right)^{2 / 3}$. We present this relation in Figure 9 as the bottom green line. In part due to the large scatter, this relation is also consistent with the observed radius evolution of starburst clusters.

The magenta dash-dotted line in Figure 9 gives the relation $\rho_{\mathrm{h}}=0.1 M_{\odot} \mathrm{pc}^{-3}$. This is an order of magnitude higher than the field density in the solar neighborhood $\left(0.01 M_{\odot} \mathrm{pc}^{-3}\right)$ (Holmberg \& Flynn 2000), and we assume this to be a minimum to the (observable) cluster density. This predicts that associations will not survive more than $20 \mathrm{Myr}$, and indeed no such a cluster has been observed.

\section{SUMMARY}

We performed a series of simulations of star-forming regions. Our calculations start with hydrodynamical simulations of turbulent molecular clouds. These simulations are continued for about one initial free-fall timescale, after which we replace gas particles with stars, adopting a local SFE (Krumholz et al. 2012). The stellar masses are selected randomly from the adopted initial mass function, and the stars receive the position and velocity of the gas particles they replace. We subsequently remove all residual gas and continue the evolution of the young emerging star cluster by means of $\mathrm{N}$-body simulations with stellar evolution.

The types of star clusters that formed in our simulations depend on the initial conditions (mass and density) of the molecular cloud. The clouds with initial conditions typical for those observed in the Milky Way $\left(10^{4} M_{\odot}\right.$ and $\left.100-1000 \mathrm{~cm}^{-3}\right)$ lead to classical open clusters. More massive clouds $\left(10^{5}-\right.$ $10^{6} M_{\odot}$ ) with the same density evolve into dense, massive clusters. These massive molecular clouds are common in starburst galaxies, but are very rare in local disk galaxies such as the Milky Way. This result is consistent with observations that young massive clusters are common in starburst galaxies, but only several have been found in the Milky Way. We argue that such massive clouds must be able to form in the Milky Way Galaxy, even though they are probably rare.

Dense, massive clusters in our simulation form from molecular clouds with a mass of $10^{6} M_{\odot}$ and a density of $\sim 1000 \mathrm{~cm}^{-3}\left(100 M_{\odot} \mathrm{pc}^{-3}\right)$, leading to a velocity dispersion of $\sim 20 \mathrm{~km} \mathrm{~s}^{-1}$. This is consistent with the relative velocity of molecular clouds observed near young massive clusters in the Milky Way, such as near NGC 3603 (Fukui et al. 2014) and Westerlund 2 (Furukawa et al. 2009; Ohama et al. 2010). We argue that massive clusters in the Milky Way can therefore not form from individual clouds, but their formation may have been initiated in cloud-cloud collisions (Furukawa et al. 2009; Ohama et al. 2010; Fukui et al. 2014).

Molecular clouds with a mass of $\sim 10^{6} M_{\odot}$ and a low density of $\sim 10 \mathrm{~cm}^{-3}\left(\sim 1 M_{\odot} \mathrm{pc}^{-3}\right)$, which follow Larson's relation, tend to form associations ("leaky clusters" in the terminology of Pfalzner 2009). These relatively low-density and massive molecular clouds form a number of small clumps. They might be detected as embedded or classical open clusters when they are young, but they evolve to less-dense clusters by gas expulsion and relaxation. After several Myr, these systems lose their clumpiness and become recognizable as associations.

In our simulations, we assumed that stars form instantaneously upon the expulsion of the residual gas (after an initial free-fall time of the molecular cloud). Our prescription for star formation is simple compared to reality, in which star formation triggers the expulsion of the residual gas by means of feedback processes. Regardless of the simplicity of our approach, we are still able to make a distinction between the formation of associations, open clusters, and massive star clusters.

The young stellar system, Sco OB2, is an assembly of associations of slightly different ages: USco, Upper Cen-Lups, and Lower Cen-Crux. A stellar system similar to Sco OB2 naturally originates in our simulations of relatively massive and low-density molecular clouds, although the age spread cannot be reproduced with our method. The relation that less-dense clusters have wider age spreads of stars is observationally and theoretically suggested (Parmentier et al. 2014).

In addition, we compared our simulations with theoretical models for cluster expansion that are due to the dynamical evolution (Gieles et al. 2011). These models satisfactorily explain the evolution in radius of simulated clusters as well as of the observed clusters.

We also found that the distribution of clusters on the massradius diagram is also limited by the density with which the dynamical timescale is equal to the cluster age. This implies that, if the cluster age is much shorter than the dynamical time, such clusters cannot be recognized as (bound) systems (Gieles $\&$ Portegies Zwart 2011). After $\simeq 20$ Myr the density of these associations drops below the background density, and they dissolve.

The gap of the radius distribution for associations and young massive clusters suggested by Pfalzner (2009) is consistent with our simulation results. Whereas young massive clusters evolve following the cluster expansion model, leaky clusters have $t_{\text {age }} \sim t_{\text {dyn }}$. With our models, the evolution of radius for observed leaky and young massive clusters are described by $r_{\mathrm{h}} / \mathrm{pc}=2.7\left(t_{\mathrm{age}} / \mathrm{pc}\right)^{2 / 3}$ and $r_{\mathrm{h}} / \mathrm{pc}=0.34\left(t_{\mathrm{age}} / \mathrm{Myr}\right)^{2 / 3}$, respectively. These are also consistent with observations. Pfalzner et al. (2014) claimed that star formation continues in embedded clusters and that after the gas expulsion they expand and become associations. Our models, however, indicate that clumpy star-forming regions are observed as a conglomerate of embedded clusters, but at a later time these systems lose their clumpiness through the expulsion of the residual gas and twobody relaxation. Because our coverage of parameter space remains limited and much is still to be uncovered, we hope to explore a much wider range of initial conditions of molecular clouds (different masses, radii, and density distributions) and other assumptions for star formation (different epochs for star formation and gradual gas removal rather than instantaneous gas expulsion).

Our results suggest that the difference in the parental molecular clouds results in the formation of various types of star clusters if we assume the same star-formation process and that the cluster-formation process does not depend on the condition of the galaxy, either normal disk or starburst.

We thank the anonymous referee for useful comments. This work was supported by JSPS KAKENHI Grant Number 26800108 and NAOJ Fellowship, the Netherlands Research 
Council NWO (grants \#643.200.503, \#639.073.803 and \#614.061.608), and the Netherlands Research School for Astronomy (NOVA). Numerical computations were partially carried out on a Cray XC30 at the Center for Computational Astrophysics (CfCA) of the National Astronomical Observatory of Japan and the Little Green Machine at Leiden Observatory.

\section{REFERENCES}

Aarseth, S. J. 1974, A\&A, 35, 237

Andersen, M., Meyer, M. R., Oppenheimer, B., Dougados, C., \& Carpenter, J. 2006, AJ, 132, 2296

Arzoumanian, D., André, P., Didelon, P., et al. 2011, A\&A, 529, L6

Bastian, N., Adamo, A., Gieles, M., et al. 2011, MNRAS, 417, L6

Bonatto, C., \& Bica, E. 2011, MNRAS, 414, 3769

Bonnell, I. A., Bate, M. R., \& Vine, S. G. 2003, MNRAS, 343, 413

Chandar, R., Whitmore, B. C., Calzetti, D., et al. 2011, ApJ, 727, 88

Drew, J. E., Busfield, G., Hoare, M. G., et al. 1997, MNRAS, 286, 538

Eisenstein, D. J., \& Hut, P. 1998, ApJ, 498, 137

Fang, M., van Boekel, R., Wang, W., et al. 2009, A\&A, 504, 461

Federrath, C. 2013a, MNRAS, 436, 1245

Federrath, C. 2013b, MNRAS, 436, 3167

Federrath, C., \& Klessen, R. S. 2013, ApJ, 763, 51

Federrath, C., Roman-Duval, J., Klessen, R. S., Schmidt, W., \& Mac Low, M.-M. 2010, A\&A, 512, A81

Figuerêdo, E., Blum, R. D., Damineli, A., \& Conti, P. S. 2002, AJ, 124, 2739

Flaherty, K. M., \& Muzerolle, J. 2008, AJ, 135, 966

Fujii, M., Iwasawa, M., Funato, Y., \& Makino, J. 2009, ApJ, 695, 1421

Fujii, M. S. 2015a, PASJ, 67, 59

Fujii, M. S. 2015b, PASJ, arXiv:1410.5540

Fujii, M. S., \& Portegies Zwart, S. 2013, MNRAS, 430, 1018

Fujii, M. S., \& Portegies Zwart, S. 2014, MNRAS, 439, 1003

Fujii, M. S., \& Portegies Zwart, S. 2015, MNRAS, 449, 726

Fukui, Y., Ohama, A., Hanaoka, N., et al. 2013, arXiv:1306.2090

Fukui, Y., Ohama, A., Hanaoka, N., et al. 2014, ApJ, 780, 36

Furukawa, N., Dawson, J. R., Ohama, A., et al. 2009, ApJL, 696, L115

Gerritsen, J. P. E., \& Icke, V. 1997, A\&A, 325, 972

Gieles, M., Athanassoula, E., \& Portegies Zwart, S. F. 2007, MNRAS, 376, 809

Gieles, M., Heggie, D. C., \& Zhao, H. 2011, MNRAS, 413, 2509

Gieles, M., \& Portegies Zwart, S. F. 2011, MNRAS, 410, L6

Girichidis, P., Federrath, C., Allison, R., Banerjee, R., \& Klessen, R. S. 2012, MNRAS, 420, 3264

Girichidis, P., Federrath, C., Banerjee, R., \& Klessen, R. S. 2011, MNRAS, 413,2741

Gürkan, M. A., Freitag, M., \& Rasio, F. A. 2004, ApJ, 604, 632

Heggie, D. C. 1975, MNRAS, 173, 729

Hénon, M. 1965, AnAp, 28, 62

Hernquist, L., \& Katz, N. 1989, ApJS, 70, 419
Heyer, M. H., \& Brunt, C. M. 2004, ApJL, 615, L45

Higuchi, A. E., Kurono, Y., Saito, M., \& Kawabe, R. 2009, ApJ, 705, 468

Hodapp, K.-W., \& Rayner, J. 1991, AJ, 102, 1108

Holmberg, J., \& Flynn, C. 2000, MNRAS, 313, 209

Horner, D. J., Lada, E. A., \& Lada, C. J. 1997, AJ, 113, 1788

Hurley, J. R., Pols, O. R., \& Tout, C. A. 2000, MNRAS, 315, 543

Hut, P. 1983, ApJL, 272, L29

Inoue, T., \& Fukui, Y. 2013, ApJL, 774, L31

Kim, W.-T., \& Ostriker, E. C. 2002, ApJ, 570, 132

King, I. R. 1966, AJ, 71, 64

Krumholz, M. R., Dekel, A., \& McKee, C. F. 2012, ApJ, 745, 69

Krumholz, M. R., \& McKee, C. F. 2005, ApJ, 630, 250

Lada, C. J., \& Lada, E. A. 2003, ARA\&A, 41, 57

Larson, R. B. 1981, MNRAS, 194, 809

Levine, J. L., Steinhauer, A., Elston, R. J., \& Lada, E. A. 2006, ApJ, 646, 1215

Luhman, K. L., Stauffer, J. R., Muench, A. A., et al. 2003, ApJ, 593, 1093

Lynden-Bell, D., \& Wood, R. 1968, MNRAS, 138, 495

Mac Low, M.-M., \& Klessen, R. S. 2004, RvMP, 76, 125

McKee, C. F., \& Ostriker, E. C. 2007, ARA\&A, 45, 565

Murray, N. 2011, ApJ, 729, 133

Nitadori, K., \& Makino, J. 2008, NewA, 13, 498

Ohama, A., Dawson, J. R., Furukawa, N., et al. 2010, ApJ, 709, 975

Ostriker, E. C., Stone, J. M., \& Gammie, C. F. 2001, ApJ, 546, 980

Parmentier, G., \& Pfalzner, S. 2013, A\&A, 549, A132

Parmentier, G., Pfalzner, S., \& Grebel, E. K. 2014, ApJ, 791, 132

Pelupessy, F. I. 2005, PhD thesis, Leiden Observatory, Leiden Univ.

Pelupessy, F. I., \& Portegies Zwart, S. 2012, MNRAS, 420, 1503

Pelupessy, F. I., van der Werf, P. P., \& Icke, V. 2004, A\&A, 422, 55

Pelupessy, F. I., van Elteren, A., de Vries, N., et al. 2013, A\&A, 557, A84

Pfalzner, S. 2009, A\&A, 498, L37

Pfalzner, S. 2011, A\&A, 536, A90

Pfalzner, S., \& Kaczmarek, T. 2013, A\&A, 559, A38

Pfalzner, S., Parmentier, G., Steinhausen, M., Vincke, K., \& Menten, K. 2014, ApJ, 794, 147

Piskunov, A. E., Kharchenko, N. V., Schilbach, E., et al. 2008, A\&A, 487, 557

Portegies Zwart, S., McMillan, S., Harfst, S., et al. 2009, NewA, 14, 369

Portegies Zwart, S., McMillan, S. L. W., van Elteren, E., Pelupessy, I., \& de Vries, N. 2013, CoPhC, 183, 456

Portegies Zwart, S. F., \& McMillan, S. L. W. 2002, ApJ, 576, 899

Portegies Zwart, S. F., McMillan, S. L. W., \& Gieles, M. 2010, ARA\&A, 48,431

Roman-Duval, J., Federrath, C., Brunt, C., et al. 2011, ApJ, 740, 120

Salpeter, E. E. 1955, ApJ, 121, 161

Spitzer, L. 1987, Dynamical Evolution of Globular Clusters (Princeton, NJ: Princeton Univ. Press)

Spitzer, L. J., \& Hart, M. H. 1971, ApJ, 164, 399

Springel, V., \& Hernquist, L. 2002, MNRAS, 333, 649

Winston, E., Megeath, S. T., Wolk, S. J., et al. 2009, AJ, 137, 4777

Wolff, S. C., Strom, S. E., Dror, D., \& Venn, K. 2007, AJ, 133, 1092

Zinnecker, H., \& Yorke, H. W. 2007, ARA\&A, 45, 481 